\title{
ADDITIONS AND CORRECTIONS TO THE “NOMECLATOR OF MEXICAN AND CENTRAL AMERICAN RUBIACEAE" OF D. H. LORENCE
}

\author{
A. BORHIDI \\ Inst. Ecology and Botany, Hungarian Academy of Sciences, H-2163, Vácrátót, Hungary; \\ E-mail: borhidi@botanika.hu \\ Research Group for Biological Adaptation, Univ. Pécs, H-7624 Pécs, Ifjúság útja 6, Hungary
}

(Received 27 September, 2000)

The article contains a number of additions and corrections - including 8 new combinations - to the Nomenclator of Mexican and Central American Rubiaceae published by D. H. Lorence, data of former publications neglected by the author, and completed by new taxonomic and nomenclatural additions as results of a thorough taxonomic study carried out in the herbaria of MEXU, MO and XAL.

Key words: Antirhea, Arachnothryx, Javorkaea, Rogiera, Rondeletia, Stenostomum, Mexico, Central America

\section{INTRODUCTION}

David H. Lorence published a very important book about the nomenclature of the plant genera and species of the family Rubiaceae occurring in Mexico and Central America. Although he knows that there are a number of publications treating some genera in different concept, accumulating new morphological, anatomical, cytological and molecular features first of all in the Rondeletieae tribe, he neglected them without mentioning any. My impression about this book is that it has not been submitted to a thorough critical reading, which would have been useful.

Therefore using the opportunity to make a detailed revising morphological study of the materials in the herbaria of the Missouri Botanical Garden (MO), MEXU and XAL complementing with results of anatomical studies obtained and partly published by the colleagues of the Botanical Department of the University of Pécs, all supporting the taxonomic and nomenclatural decisions listed below. 


\section{Additions and corrections}

Antirhea Commers. et Juss., Gen. Pl., p. 204. (1789), based on the type Antirhea borbonica J. F. Gmel. does not occur in Mexico and Central America. All the neotropical taxa classified into the Antirhea belong to different endemic neotropical genera, mostly into the genus Stenostomum C. F. Gaertn. (Chaw and Darwin 1992, Borhidi and Fernandez 1995a, b).

Antirhea aromatica Cast.-Campos et Lorence, Ann. Mo. Bot. Gard. 72: 268. Fig. 1 (1985). = Stenostomum aromaticum (Cast.-Campos et Lorence) Borhidi in Borhidi and Fernandez, Acta Bot. Hung. 38: 160. 1993-94 (publ. 1995). Type: Mexico: Veracruz, Mpio Jalcomulco, ca $10 \mathrm{~km}$ al $\mathrm{S}$ de Apazapan, Barranca de Monterrey, al S de Cuetzalan, entre Cuetzalan y Apazapan, alt. 350 m, 17.09.1983. Holotype: XAL!, isotypes: MEXU!, MO!, XAL!

Arachnothryx Planch., Fl. Serres Jard. 5: tab. 442. (1849). Type: Arachnothryx leucophylla (Kunth) Planch. designated by Borhidi (1982).

Capsule oval or oblong, septicidally dehiscent; seeds rhomboidal to triangular, compressed, wingless (roundly winged only in A. galeottii) or not caudate with lateral insertion, the testa deeply foveolate and rugulose with sunken pentagonal or hexagonal cells; perpendicular cell walls thick and doubled without ornamentation, horizontal cell walls deeply concavious, without or with obsolete thickenings. Orifice of the corolla open, naked, without a thickened callosity or hairs; corolla tube pubescent within basal portion; corolla lobes 4; calyx lobes 4; disc glabrous sunken below sinus at the base of the calyx lobes; calyx tube obviously manifested, placenta ovate with central punctiform insertion to the septum, pubescence of hypanthium and corolla tube without usually pannose or arachnoid pubescent; pollen grains oblong-elliptic, 3-colporate, exine thick, sparsely foveolate with regular tight and shallow foveoles, style long naked, shortly bilobate at the tip; basic chromosome number $x=9$.

About 75 species in Mexico, Central America and N of South America.

Arachnothryx acuminata (Oerst. ex Standl.) Borhidi, Acta Bot. Hung. 35: 310. (1989). Basionym: Rondeletia scabra Hemsl., Diagn. Pl. Nov. Mexic., p. 29. (1879). = Javorkaea scabra (Hemsl.) Borhidi, comb. nova hoc loco. 
Arachnothryx aetheocalymna (Donn.Sm.) Borhidi, Acta Bot. Hung. 28: 70. (1982). Basionym: Rondeletia aetheocalymna Donn.Sm., Bot. Gaz. 42: 298. (1906).

Arachnothryx affinis (Hemsl.) Borhidi, Acta Bot. Hung. 38: 139. 1993-94 (publ. 1995). Basionym: Rondeletia affinis Hemsl., Diagn. Pl. Nov. Mexic., p. 28. (1879). = Arachnothryx buddleioides (Benth.) Planch.

Arachnothryx albida (Lundell) Borhidi, Acta Bot. Hung. 35: 309. (1989). Basionym: Rondeletia albida Lundell, Wrightia 5: 323. (1976). = Arachnothryx laniflora (Benth.) Planch.

Arachnothryx aspera (Standl.) Borhidi, Acta Bot. Hung. 28: 68. (1982). Basionym: Rondeletia aspera Standl., N. Amer. Fl. 32: 54. (1918).

Specimens examined: Costa Rica: Puntarenas, Cantón de Puntarenas, $10 \mathrm{~km} \mathrm{SW}$ of Santa Elena on road from Monteverde, to Interamerican Hwy, coord.: $10^{\circ} 15^{\prime} \mathrm{N}, 84^{\circ} 50^{\prime} \mathrm{W}$, alt. 750-850 m, leg.: W. Haber 10312, 26.12.1990. (CR, MO). - Puntarenas, Cantón de Puntarenas, Monteverde, bajo Tigre trail, pacific slope, premontane moist forest, coord.: $10^{\circ} 17^{\prime}$ N, $84^{\circ} 48^{\prime}$ W, alt. 1100-1200 m, leg.: W. Haber 10954. (MO). - Puntarenas, Cantón de Puntarenas, sendero a la Catarata por el rio, sendero Miguel Leitón. Coord.: $10^{\circ} 16^{\prime} 20^{\prime \prime} \mathrm{N}$, 8449'30" W, alt. 1100 m, leg.: Z. Fuentes, M. Zamora \& E. Lepis 245, 11.03.1993. (MO). Puntarenas, Monteverde area, near to Rio San Luis, just $S$ of Monteverde, in woods along the river, coord.: $10^{\circ} 16^{\prime} \mathrm{N}, 84^{\circ} 48^{\prime} \mathrm{N}$, alt. 1000-1200 m, leg.: B. Hammel \& W. Haber 13929, 18.06.1985. (MO). - Puntarenas, Monteverde, Guasimal, river valley below Monteverde on pacific slope, moist forest, leg.: W. Haber \& E. Bello 4314, 17.01.1986. (MO). - Puntarenas, Monteverde, pacific slopes on Guasimal, 900 m, Quebrada Cambronero, leg.: W. A. Haber 1218, 13.01.1965. (MO). - Puntarenas, Monteverde, slopes below Monteverde from $1000 \mathrm{~m}$, leg.: W. Haber 424, 10.01.1981. (MO). - Puntarenas, Monteverde, San Luis, valley below community, premontane moist forest on pacific slope. Alt. 1200 m, leg.: W. A. Haber \& E. Bello 4040, 30.12.1985. (MO). - Puntarenas, Reserva Biológica Monteverde, sendero San Luis, coord.: $10^{\circ} 18^{\prime} \mathrm{N}, 84^{\circ} 48^{\prime} \mathrm{W}$, alt. $1450 \mathrm{~m}$, leg.: W. Haber, E. Bello \& W. Gove 6513. (CR, MO). - San José, Cantón de Acosta, Cerros de Escazú, Agua Blanca, Alto El Guabal, bosque secundario por el camino a Quebrada Mina. Coord.: 949'20” N, $84^{\circ} 11^{\prime}$ W. Leg.: J. F. Morales \& M. Barrantes 2362, 12.02.1994. (MO). - San José, Cantón de Mora, ciudad Colón, zona protectora El Rodeo, por bajo Morales, coord.: 09 $54^{\prime}$ N, $84^{\circ} 16^{\prime} \mathrm{W}, 900 \mathrm{~m}$, leg.: Q. Jimenez 957, 06.03.1991. (MO). - San José, Cantón de Mora, entre Hacienda El Rodeo y Piedras Negras, coord.: 9 $55^{\prime} 40^{\prime \prime}$ N, 8417'10" W, alt. 700 m, leg.: B. Hammel, I. Hammel \& P. Rivera 18687, 25.12.1992. (MO). - San José, Cantón de Mora, valle de Tárcoles, Alto Tigre, al E de Piedras Negras. Coord.: 954'50" N, 84¹7'40" W, alt. 800 m, leg.: E. Lepis 174, 14.02.1994. (MO). - San José, elevado de Rios Corrogres, Santa Ana, alt. 900 m, leg.: A. Jimenez M. 2751, 18.01.1965. (MO). - Santiago de Ramón, Finca Barranca, alt. 900 m, leg.: I. A. Chacón \& G. Herrera 20771, 06.01.1984. (Hb. Nacional San José, MO). 
Arachnothryx atravesadensis (Lorence) Borhidi, Acta Bot. Hung. 38: 139. 1993-94 (publ. 1995). Basionym: Rondeletia atravesadensis Lorence, Novon 1: 137. (1991).

Arachnothryx belizensis (Standl.) Borhidi, Acta Bot. Hung. 28: 70. (1982). = Rondeletia belizensis Standl.

Arachnothryx bertieroides (Standl.) Borhidi, Acta Bot. Hung. 28: 70. (1982). Basionym: Rondeletia berterioides Standl., Publ. Field. Col. Mus., Bot. Ser., p. 267. (1929).

Specimens examined: Costa Rica: Limón, bosque Aguas Arriba de la Catarata, del Rio Sandbogs Dalamanca, alt. 200 m, leg.: J. Gómez Laurito 9334, 23.04.1983. (MO). - Limón, evergreen forest near the Rio Catarata, Rio Sandbogs, in the hills, between Bribrí on the Rio Sixaola and the Caribbean coastal plain. Coord.: $9^{\circ} 37^{\prime}$ N, $82^{\circ} 49^{\prime} \mathrm{W}$, alt. 50-100 m. (MO). Limón, Tortuguero, Cantón along road of Bribrí and Suretka, alt. 100-200 m, leg.: K. Barringer, E. Cristenson \& J. Gómez Laurito 3523, 06.07.1983. (MO). - Puntarenas, Cantón de Golfito, near and along trail leading from $\mathrm{km} 3$ Torres to Quebrada Culebra, tributary of Rio Sorpresa, ca $2.5 \mathrm{~km}$ NE of Golfito. Coord.: $8^{\circ} 39^{\prime} 00^{\prime \prime} \mathrm{N}, 83^{\circ} 09^{\prime} 00^{\prime \prime} \mathrm{W}$, alt. 300-380 m, leg.: M. Grayum, B. Hammel, F. Martín \& U. Chavarría 10094, 01.02.1992. (CR, MO). Puntarenas, Cantón de Osa, Rancho Quemado, sector O Sierpe, coord.: $8^{\circ} 40^{\prime} 30^{\prime \prime} \mathrm{N}$, 83³5’00" W, alt. 250 m, leg.: J. Marín 495, 16.08.1992. (MO). - Puntarenas, Cantón de Osa, Rancho Quemado, Reserva Forestal Golfo Dulce, coord.: 842'00" N, 833' $40^{\prime \prime}$ W, alt. 300 m, leg.: B. Hammel, J. Marín \& F. Quesada 18305, 11.07.1999. (CR, MO).

Arachnothryx bourgaei (Standl.) Borhidi, Acta Bot. Hung. 28: 69. (1982). Basionym: Rondeletia bourgaei Standl., N. Amer. Fl. 32: 56. (1918). = Arachnothryx laniflora (Benth.) Planch.

Arachnothryx brenesii (Standl.) Borhidi, Acta Bot. Hung. 33: 301. (1987). Basionym: Rondeletia brenesii Standl., Publ. Field Mus. Nat. Hist., Bot. Ser., 18: 1370. (1938).

Arachnothryx brevicorolla (J. H. Kirkbr.) Borhidi, Acta Bot. Hung. 28: 69. (1982). Basionym: Rondeletia salicifolia Dwyer et Hayden subsp. brevicorolla J. H. Kirkbr., Ann. Mo. Bot. Gard. 55: 383. (1968). = Arachnothryx bertieroides (Standl.) Borhidi.

Arachnothryx buddleioides (Benth.) Planch., Fl. Serres Jard. 5: tab. 442. (1849). Basionym: Rondeletia buddleioides Benth., Pl. Hartweg., p. 69. (1840). 
Arachnothryx calycosa (Donn.Sm.) Borhidi, Acta Bot. Hung. 28: 70. (1982). Basionym: Rondeletia calycosa Donn.Sm., Bot. Gaz. 56: 59. (1913).

Specimens examined: Costa Rica: Guanacaste, Parque Nacional de Guanacaste, Estación Cacao Liberia, coord.: $10^{\circ} 55^{\prime} 45^{\prime \prime} \mathrm{N}, 8^{\circ} 28^{\prime} 15^{\prime \prime}$ W, alt. $1100 \mathrm{~m}$, leg.: Carlos Chávez 285, 28.10.1990. (MO). - Heredia, $2.5 \mathrm{~km}$ N of Varablanca, in vicinity of Rio Paz Grande, alt. 1270-1350 m, leg.: T. B. Croat 36038, 22.06.1976. (MO). - Heredia, Varablanca de Sarapiqui, $\mathrm{N}$ slope of the Cordillera Central between Poás and Barba volcanoes, alt. $1585 \mathrm{~m}$, leg.: A. F. Skutch 3357, 10.1937. (MO). (Rondeletia torresii, det. Standley). - Heredia, cerca del Rio Parablanco, alt. 1500 m, leg.: R. W. Lent 3154, 08.07.1973. (MO). - en la frontera de las provincias Heredia y San José, SW sobre el Rio Parablanco, banks of road on open pastures on the lower slopes of Cerro Zurqui, alt. 1400-1650 m, leg.: J. and C. Utley 1274, 15.10.1971. (CR, F, MO). - Heredia, on slopes of Cerro Zurqui, above del Rio Parablanco, low forest and wet upland pastures, leg.: J. Taylor 17491, 22.12.1977. (MO). - border of Heredia and San José, SE slopes of Cerro Zurqui, at termination of calle Zurqui, alt. 1800-2000 m, leg.: F. Almeda \& K. Nakai 3710, 19.01.1978. (MO). - border of Heredia and San José, Rio Parablanco, pacific trails, Cerro Zurqui, coord.: 10,03 N, 84,01 W, alt. 1600-1800 m, leg.: W. Burger, C. Baker \& J. Utley 9349, 21.10.1975. (MO). - San José, Z. P. Cerros Torrubal faldas del Cerro Bares, coord.: $9^{\circ} 47^{\prime} 30^{\prime \prime} \mathrm{N}, 84^{\circ} 28^{\prime} 30^{\prime \prime} \mathrm{W}$, alt. $1600 \mathrm{~m}$, leg.: Q. Jimenez, L. Zuniga \& G. Juarela 120, 06.10.1990. (CR, MO).

Arachnothryx capitellata (Hemsl.) Borhidi, Acta Bot. Hung. 28: 69. (1982). Basionym: Rondeletia capitellata Hemsl., Diagn. Pl. Nov. Mexic., p. 28. (1879).

Specimens examined: Mexico: Oaxaca, Distr. Mixe, $10 \mathrm{~km}$ al NE de la desviación a Totontepec, sobre la carretera Tamazulpán-Zacatepec, leg.: L. A. Rico, R. C. Torres \& R. T. Cedillo 370. (MO). - Oaxaca, Distr. Mixe, 2 km sobre la Terracería Totontepec, Via Alta, leg.: A. Mendoza \& R. Torres 13, 18.01.1986. (MO). - Oaxaca, Distr. Mixe, $9 \mathrm{~km}$ a la entrada del camino a Via Alta, entronque que se encuentra aprox. a $10 \mathrm{~km}$ de Totontepec, en la Barranca, leg.: R. C. Torres, L. Cortés A. \& C. Martinez 2950. (MO). - Oaxaca, Distr. Mixe, 9 $\mathrm{km}$ de la entrada al camino a Via Alta, entronque que se encuentra aprox. a $10 \mathrm{~km}$ de Totontepec, en la Barranca, leg.: R. Torres, L. Cortés A. \& C. Martinez 7958, 13.12.1985. (MO). - Oaxaca, Distr. Mixe, Mpio. Totontepec, ca $1900 \mathrm{~m}$, coord.: $17^{\circ} 15^{\prime} \mathrm{N}, 96^{\circ} 02^{\prime} \mathrm{W}$, leg.: J. L. Rey \& G. J. Martin 1164, 12.10.1988. (MO). - Oaxaca, Distr. Mixe, Mpio. Totontepec, ca 1900 m, coord.: $17^{\circ} 15^{\prime}$ N, $96^{\circ} 02^{\prime}$ W, leg.: J. L. Rey \& G. J. Martin 851, 01.11.1987. (MO). Oaxaca, Distr. Via Alta, $15 \mathrm{~km}$ del entronque San Andrés Yaja, SE de Via Alta, leg.: R. C. Torres \& R. T. Cedillo 150, 17.03.1982. (MO). - Oaxaca, Distr. Via Alta, 6 km N del entronque de Mixititlán y Totontepec, hacía San Andrés Yaja, leg.: R. C. Torres \& R. T. Cedillo 490, 19.05.1982. (MO). - Oaxaca, Distr. Via Alta, Camillo Axiacui, a Talía de Castro, 18 al S de Talía por camino, leg.: D. H. Lorence 4095, 19.04.1982. (MO). - Oaxaca, Distr. Via Alta, desviación a Oaxaca por Mixes, $3.5 \mathrm{~km}$ al S de San Andrés Yaja, leg.: R. C. Torres \& R. T. Cedillo 2070, (MO). - Oaxaca, $6.7 \mathrm{~km}$ al SE de la desviación a Oaxaca por Mixes, la desviación esta a $3.5 \mathrm{~km}$ al S de San Andrés Yaja, distr. de Via Alta, alt. $2020 \mathrm{~m}$, leg.: R. Torres \& R. Cedillo 2058, (MO). - Oaxaca, Distr. Via Alta, Sierra de Juárez, camino de Caljualpán a Talía, al 10 km N de la desviación a Yalina, leg.: D. H. Lorence 4228, 31.05.1983. (MO). 
Arachnothryx chaconis (Lorence) Borhidi, Acta Bot. Hung. 38: 140. 1993-94 (publ. 1995). Basionym: Rondeletia chaconii Lorence in W. C. Burger and C. M. Taylor, Fieldiana, Bot., nov. ser., 33: 296. (1993). = Javorkaea chaconii (Lorence in W. C. Burger and C. M. Taylor) Borhidi, comb. nova hoc loco.

Arachnothryx chiapensis (Brandegee) Borhidi, comb. nova hoc loco. Basionym: Otocalyx chiapensis Brandegee, Univ. Calif. Publ. Bot. 6: 69. (1914). Synonym: Rondeletia brandegeeana Lorence, Novon 1: 138. (1991). The new name created by Lorence is illegitimate, because the epithets chiapensis and chiapasensis cannot be considered as homonyma.

Arachnothryx chinajensis (Standl. et Steyerm.) Borhidi, Acta Bot. Hung. 33: 301. (1987). Basionym: Rondeletia chinajensis Standl. et Steyerm., Publ. Field. Mus. Nat. Hist., Bot. Ser., 23: 254. (1943).

Arachnothryx chiriquiana (Lorence) Borhidi, Acta Bot. Hung. 40: 15. 1996-97 (publ. 1999). Basionym: Rondeletia chiriquiana Lorence, Novon 4 : 129-132. (1994).

Arachnothryx cooperi (Standl.) Borhidi, Acta Bot. Hung. 28: 69. (1982). Basionym: Rondeletia cooperi Standl., Publ. Field Mus. Nat. Hist., Bot. Ser., 4: 267. (1929).

Specimen examined: Costa Rica: San José, Estación Carrillo, 700-450 m de la fila al Canón del Rio Sucio, leg.: I. A. Chacón \& G. Herrera 1689, 12.11.1983. (MO).

Arachnothryx cordovana (Standl. et Steyerm.) Borhidi, Acta Bot. Hung. 33: 301. (1987). Basionym: Rondeletia cordovana Standl. et Steyerm., Publ. Field Mus. Nat. Hist., Bot. Ser., 23: 26. (1943). = Arachnothryx chiapensis (Brandegee) Borhidi.

Arachnothryx costaricensis (Standl.) Borhidi, Acta Bot. Hung. 28: 70. (1982). Basionym: Rondeletia costaricensis Standl., N. Amer. Fl. 32: 61. (1918).

Specimens examined: Costa Rica: Prov. Alajuela, about $11 \mathrm{~km} \mathrm{~N}$ of Rio Naranjo, along road to Upala, alt. 400-500 m. Leg.: J. \& C. Utley 5361, 08.07.1976. (MO). - Prov. Alajuela, between Canas and Upala, 4 km NNE de Bijagua, slopes leading into Rio Zapote alt. $400 \mathrm{~m}$. Leg.: T. B. Croat 36301, 24.06.1976. (MO). - Prov. Alajuela, Cantón de San Ramón, Cordillera de Tilarán, Estación Rio San Lorenzo, sendero al volcán Muerto, coord.: 10¹2’55” N, 84³6'25" W, alt. 1100 m. Leg: E. Quesada 30, 26.04.1993. (MO). - Prov. Alajuela, Reserva

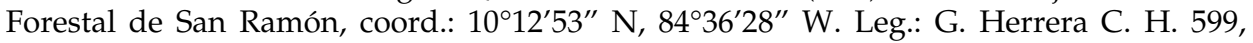


02.05.1987. (MO). - Prov. Alajuela, San Ramón to Santa Rita de San Carlos, home route a Lagitos km $311^{\circ} 20^{\prime} \mathrm{N}, 84^{\circ} 31^{\prime} \mathrm{W}$, alt. 600 m. Leg: B. Hammel, G. de Nevers \& C. Gomez 15301, 01.06.1986. (MO). - Prov. Alajuela, La marina de San Carlos, alt. 500-700 m. Leg.: N. Zamora, A. Guevara \& O. Sandoval 1502, 12.08.1988. (MO). - Prov. Alajuela, Santa Maria Parque Nacional, $7 \mathrm{~km}$ E of Coloured House at the junction of road to Hacienda Santa Maria, alt. 600 m. coord.: $10^{\circ} 48^{\prime}$ N, 85¹6’ W. Leg.: R. Liesner 5148, 08.02.1978. (MO). - Prov. Alajuela, Villa Quesada, Cantón San Carlos, leg.: A. Smith 1625, alt. 850 m. (MO). - Prov. Alajuela, Villa Quesada, Cantón San Carlos, alt. 1750 m. Leg.: A. Smith 1948. (MO). - Prov. Alajuela, Villa Quesada, Cantón San Carlos, leg.: A. Smith 2520. (MO). - Guanacaste, Parque Rincón de la Vieja, Hacienda Santa Maria de la Planta Hidroeléctrica, sigiendo el canal hasta la Quebrada de Provisión, coord.: 1048’ N, 85¹9’ W, alt. 800-900 m. Leg.: G. Herrera 820, 17.10.1987. (MO). - San José, Santa Rosa de Puriscal. Leg.: L. J. Poveda 439, 17.01.1973. (MO). - San José, Z. P. Santa Rosa de Puriscal, coord.: 941'50" N, 84²3'30” W, alt. 450 m. Leg.: J. F. Morales 381, 13.08.1992. (MO). Honduras: 1-2 km NNW of Cabeza del Negro, coord.: $15^{\circ} 29^{\prime} \mathrm{N}, 87^{\circ} 27^{\prime}$ W, alt. 250 m. Leg.: D. L. Haslett \& A. E. Brandt 8098, 26.04.1994. (MO). - Atlantida, Campamento Quebrada Grande, ca $10 \mathrm{~km} \mathrm{~W} \mathrm{of} \mathrm{La} \mathrm{Ceiba,} \mathrm{at}$ base of slopes of Pico Bonito, coord.: $15^{\circ} 42^{\prime}$ N, 86 $51^{\prime}$ W, alt. 80-150 m. Leg.: R. L. Liesner 26105, 09.05.1993. (MO). - Atlantida, Campamento Quebrada Grande, ca $10 \mathrm{~km}$ NW of La Ceiba, at base of N slopes of Pico Bonito, alt. 80-100 m. Leg.: R. L. Liesner 26214, 11.05.1993. (MO). - Prov. Atlantida, ravines E of Texiquat River, 1-2 km SW of aldea La Aurora, coord.: $15^{\circ} 30^{\prime}$ N, 87²6’ W, alt. 200-500 m. Leg.: D. L. Haslett, A. E. Brandt \& R. Zuniga 8018, 09.04.1994. (MO). - Yoro, Guán, Cascada del Rio Guán, E slopes of the Rio Guán, Guán waters. Coord.: $15^{\circ} 29^{\prime} 40^{\prime \prime}$ N, 87²7'16" W, alt. 400-440 m. Leg.: T. Hawkins \& M. Merello 780, 21.04.1995. (MO).

Arachnothryx darcyi (Dwyer) Borhidi, Acta Bot. Hung. 28: 70. (1982). Basionym: Rondeletia darcyi Dwyer, Ann. Mo. Bot. Gard. 67: 467. (1980). = Arachnothryx secunda (Standl.) Borhidi.

Arachnothryx dariensis (Standl.) Borhidi, Acta Bot. Hung. 28: 68. (1982). Basionym: Rondeletia dariensis Standl., N. Amer. Fl. 32: 53. (1918).

Specimen examined: Panama: Edo, Darién, W slope of Cerro Pirre above Renare Camp and park shelter, coord.: $8^{\circ} 00^{\prime} \mathrm{N}, 77^{\circ} 45^{\prime} \mathrm{W}$, alt. 550-775 m, leg.: G. McPherson 12644 , 28.07.1988. (MO).

Arachnothryx deamii (Donn.Sm.) Borhidi, Acta Bot. Hung. 28: 68. (1982). Basionym: Bouvardia deamii Donn.Sm., Bot. Gaz. 49: 455. (1910). = Rondeletia deamii (Donn.Sm.) Standl.

Arachnothryx dwyeri (Lorence) Borhidi, Acta Bot. Hung. 38: 140. 1993-94 (publ. 1995). Basionym: Rondeletia dwyeri Lorence, Novon 1: 139. (1991). 
Arachnothryx edwardsii (Standl.) Borhidi, Acta Bot. Hung. 33: 301. (1987). Basionym: Rondeletia edwardsii Standl., Trop. Woods 37: 31. (1934). = Rogiera edwardsii (Standl.) Borhidi, comb. nova hoc loco.

Arachnothryx evansii (Lorence) Borhidi, Acta Bot. Hung. 40: 15. 1996-97 (publ. 1999). Basionym: Rondeletia evansii Lorence, Novon 7: 52-54. (1997).

Specimens examined: Honduras: Prov. Lempira, Parque Nacional de Celaque, sendero entre la Planta Eléctrica y la primera casa abandonada de Don Tomás. Coord.:

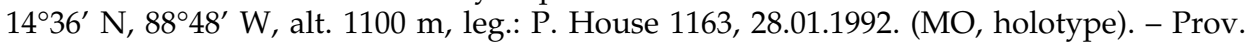
Lempira, Don Tomás, Parque Nacional de Celaque, leg.: T. Hawkins 65, 11.11.1991. (MO).Prov. Lempira, along trail from Camp Don Thomas to Camp Naranjo, near Camp Naranjo, straight line $1 \mathrm{~km}$ WSW of Gracias, Parque Nacional de Celaque, coord.: $14^{\circ} 33^{\prime} \mathrm{N}, 88^{\circ} 40^{\prime} \mathrm{W}$, alt. 2540 m, leg.: R. Evans 1188, 12.02.1993. (MO). - Prov. Lempira, Montana de Celaque, arriba de la Planta Eléctrica, alt. 2100 m, leg.: D. Haslett 2288, 18.11.1974. (MO).

Arachnothryx falciformis (Lundell) Borhidi, Acta Bot. Hung. 35: 310. (1987). Basionym: Rondeletia falciformis Lundell, Wrightia 5: 324. (1976). = Arachnothryx heteranthera (Brandegee) Borhidi.

Arachnothryx galeottii (Standl.) Borhidi, Acta Bot. Hung. 28: 70. (1982). Basionym: Rondeletia galeottii Standl., N. Amer. Fl. 32: 59. (1918).

Specimens examined: Mexico: Oaxaca, Mpio. de Hidalgotitlán, Tres Picos, a $16 \mathrm{~km}$ del campamento Hermanos Cedillo, alt. 150 m, leg.: B. Ortiz \& Martiniano 134, 29.04.1975. (MO). - Oaxaca, Mpio. Matías Romero, Zona de Uxpanapa, en la frontera de Veracruz y Oaxaca, al $3.5 \mathrm{~km}$ del Aserradero La Floresta. 16-18 km al S de Esmeraldaa lo largo del Rio Verde, coord.: $17^{\circ} 02^{\prime} 30^{\prime \prime} \mathrm{N}, 94^{\circ} 47^{\prime} \mathrm{W}$, alt. $200 \mathrm{~m}$, leg.: T. Wendt \& Villalobos 3160, 08.04.1981. (MO). - Oaxaca, Mpio. Santa María Chimalapa, Arroyo Rancho Viejo ca 3-4 km al E de Santa María, leg.: H. Hernandez G. \& C. Gonzalez 1846, 21.11.1985. (MO). - Oaxaca, Mpio. Santa María Chimalapa, cabezada del Rio Escolapa, ca $15 \mathrm{~km}$ al S de Santa María, coord.: 16 $50^{\prime}$ N, 944' W, alt. 400 m, leg.: H. Hernandez G. \& C. Gonzalez L. 1698, 21.10.1981. (MO). - Oaxaca, Mpio. Santa María Chimalapa, Canada con arroyo cerca del Rio Negro, vereda al paso La Ciruela, ca 5-7 km al S de Santa María, leg.: H. Hernandez G. 1071, 03.04.1985. (MO, fl.!). - Oaxaca, Mpio. Santa María Chimalapa, Cerro de la Fortuna, alt. 1000 $\mathrm{m}, \mathrm{SE}$ de la Sierra Tres Picos, al lado N de la acabezera del Rio Verde. Leg.: T. Wendt \& H. Hernandez G. 5712, 29.04.1987. (MO). - Oaxaca, Mpio. Santa María Chimalapa, Filo de 1 Cerro en la vereda de Mal Paso, Yucuipac del Rio Verde, leg.: H. Hernandez G. 2767, 29.10.1985. (MO). - Oaxaca, Mpio. Santa María Chimalapa, Rio Verde, ca $7 \mathrm{~km}$ al N de Santa María, coord.: $16^{\circ} 58^{\prime}$ N, $94^{\circ} 41^{\prime}$ W, leg.: H. Hernandez G. 1257, 13.07.1985. (MO). - Oaxaca. Mpio. Matias Romero, $2 \mathrm{~km}$ al SE del Aserradero La Floresta, sobre camino a Rio Verde, coord.: $17^{\circ} 03^{\prime} \mathrm{N}, 94^{\circ} 47^{\prime} \mathrm{W}$, alt. $250 \mathrm{~m}$, leg.: T. Wendt et al. 3298, 25.05.1981. (MO). - Veracruz, de Bastonal a Sierra Santa Marta en el camino ca $14 \mathrm{~km}$ del Lago Catemaco, leg.: A. Gentry \& E. Lot 32437, 29.05.1981. (MO). -Veracruz, De Catemaco a Sontecomapan a $5 \mathrm{~km}$ al N del entronque a Laguna Catemaco, coord.: $18^{\circ} 29^{\prime} \mathrm{N}, 95^{\circ} 04^{\prime} \mathrm{W}$, alt. $500 \mathrm{~m}$, leg.: M. Nee \& J. I. Calzada 22527, 31.10.1981. (MO). - Veracruz, Mpio. Catemaco, Cumbres de Bastonal, leg.: 
S. Ponce C. 296, 23.04.1974. (MO). - Veracruz, Cerro del Villa, Catemaco, alt. 700 m, leg.: J. I. Calzada 2047, 29.05.1972. (MO). - Veracruz, Est. Biol. Los Tuxtlas, alt. 200-500 m, Cerro Villa, leg.: A. Gentry \& E. Lot 32240, 21.05.1981. (MO). - Veracruz, Est. Biol. Los Tuxtlas, Lote 67, leg.: G. I. Manriquez 1743, 13.06.1984. (MO). - Veracruz, Est. Biol. Los Tuxtlas, leg.: C. León et al. 158, 03.08.1986. (MO). - Veracruz, Est. Biol. Los Tuxtlas, Laguna Escondida 3 km al NO de la Estación, leg.: S. Sinaca Colin 192, 13.08.1985. (MO). - Veracruz, Est. Biol. Los Tuxtlas, Laguna Safacal, 2.5 km N al Estación, leg.: S. Sinaca Colin, 636, 09.04.1986. (MO). Veracruz, Mpio San Andrés Tuxtla, cerca al Estación Biológica Tropical, leg.: G. I. Manriquez 554, 21.04.1983. (MO). - Veracruz, Mpio. San Andrés Tuxtla, Los Tuxtlas, Lote 67, el Villa, Estación Biologica Tropical, leg.: G. I. Martinez 780, 29.07.1983. (MO). Veracruz, Mpio. San Andrés de Tuxtla, Los Tuxtlas. leg.: M. Calderón 3063, 04.07.1970. (MO). - Veracruz, Mpio. San Andrés Tuxtla, leg.: S. Sinaca Colin 612, 16.04.1986. (MO). Veracruz, Mpio Hidalgotitlán, 2-5 km en el camino del plan arroyo a Rio Alegre, coord.: $17^{\circ} 15^{\prime} \mathrm{N}, 94^{\circ} 35^{\prime}$ W, leg.: J. Dorantes et al. 2999. (MO). - Veracruz, Mpio. Hidalgotitlán, 2 km camino plan de arroyo a Rio Alegre, coord.: $17^{\circ} 15^{\prime} \mathrm{N}, 94^{\circ} 40^{\prime} \mathrm{W}$, alt. $140 \mathrm{~m}$, leg.: J. Dorantes et al. 3037. (MO). - Veracruz, Mpio Hidalgotitlán, Rio Soloxuchil, al SO del campamento Hermanos Cedillo, leg.: S. Ponce C. 245, 02.04.1974. (MO). - Veracruz, Mpio. Hidalgotitlán,

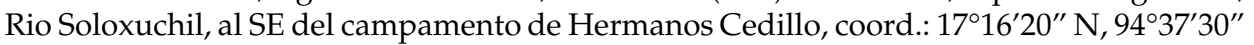
W, alt. 150 m, leg.: M. Vazquez 279A, 02.04.1974. (MO).

Arachnothryx ginetteae (Lorence) Borhidi, Acta Bot. Hung. 38: 140. 1993-94 (publ. 1995). Basionym: Rondeletia ginetteae Lorence, Novon 1: 141. (1991).

Specimen examined: Mexico: Oaxaca, Distr. de Tuxtepec, ca $10 \mathrm{~km}$ al S de Valle Nacional, alt. 750 m, leg.: D. H. Lorence \& R. T. Cedillo 2973, 06.12.1980. (MEXU, MO, type).

Arachnothryx gonzaleoides (Standl.) Borhidi, Acta Bot. Hung. 33: 302. (1987). Basionym: Rondeletia gonzaleoides Standl., Publ. Field Col. Mus., Bot. Ser., 8: 160. (1930).

Specimens examined: Guatemala: Dept. Baja Verapaz, Nino Perdido, bordering Rio San José, 8 km N in high forest. Leg.: C. L. Lundell, \& E. Contreras 20989, 26.05.1977. (MO). Mexico: Chiapas, Mpio. Ixtacomitán, lower montane rain forest along stream above Ixtacomitan. alt. 455 m, leg.: D. E. Breedlove \& F. Almeda 56779, 07.01.1982. (MO). Chiapas, Mpio. Ixtacomitán, lower montane rain forest, ridge above Ixtacomitán, alt. $300 \mathrm{~m}$, leg.: D. E. Breedlove 35055, 10.05.1973. (MO). - Chiapas, Mpio. Peltalcingo, steep slope of Ahkúlbal Nab, above Peltalcingo, alt. 1700 m, leg.: D. E. Breedlove 50397, 28.03.1981. (MO). - Chiapas, Mpio. Solosuchiapa, lower montane rain forest 3-5 km above Solosuchiapa along road to Tapilula, alt. 450 m, leg.: D. E. Breedlove 26469, 26.07.1972. (MO). - Chiapas, Mpio. Tila, Finca Morelia, leg.: A. Mendez Ton 6760, 01.10.1983. (MEXU, MO). - Chiapas, Mpio. Tila, pié del Cerro Takábana, alt. 800 m, leg.: A. Mendez Ton 5990, 03.05.1983. (MEXU, MO). - Chiapas, Mpio. Tila, pié del Cerro Takábana, alt. 800 m, leg.: A. Mendez Ton 5766, 25.03.1983. (MEXU, MO). - Veracruz, Mpio. Minatitlán, orilla del Rio Uxpanapaarriba del poblado Uxpanapa, coord.: $17^{\circ} 13^{\prime} \mathrm{N}, 9^{\circ} 12^{\prime} \mathrm{W}$, alt. $120 \mathrm{~m}$, leg.: T. Wendt et al. 3324, 26.05.1981. (MEXU, MO). 
Arachnothryx gracilis (Hemsl.) Borhidi, Acta Bot. Hung. 28: 69. (1982). Basionym: Rondeletia gracilis Hemsl., Diag. Pl. Nov. Mexic., p. 53. (1880).

Specimen examined: Guatemala: Dept. Alta Verapaz, Cobán, alt. 1600 m, leg.: H. von Türckheim 1738, 03.1908. (MO).

Arachnothryx gracilispica (Standl.) Borhidi, Acta Bot. Hung. 33: 302. (1987). Basionym: Rondeletia gracilispica Standl., Publ. Field Mus. Nat. Hist., Bot. Ser., 8: 342. (1931).

Specimens examined: Mexico: Oaxaca, Distr. Ixtlán $21.4 \mathrm{~km}$ al S del Valle Nacional sobre carretera a Oaxaca, alt. 1140 m, leg.: T. Wendt, E. J. Lott \& J.Garcia P. 2273, 28.11.1979. (MO). - Oaxaca, Distr. Ixtlán, Mpio. Comaltepec, La Esperanza, alt. ca 1600 m, coord.: 17³7' N, 96²1' W, leg.: E. Lopez Luna 148, 08.12.1987. (MO). - Oaxaca, Distr. Ixtlán, Mpio. Comaltepec, Puerto Eligio, alt. ca 700 m, coord.: $17^{\circ} 71^{\prime}$ N, $96^{\circ} 17^{\prime}$ W, leg.: E. Lopez García 78 , 14.12.1987. (MO). - Oaxaca, Distr. Ixtlán, Sierra de Juárez, ruta 175 Tuxtepec a Oaxaca a 2.5 km al NE de Puerto Eligio, leg.: D. H. Lorence \& R. T. Cedillo 4247, 01.06.1983. (MEXU, MO). - Oaxaca, entre Puerto Eligio y Comaltepec, km 146 carr. Tuxtepec-Oaxaca Cerro Metate, Sierra Juárez, alt. 710 m, leg.: G. Martinez C. 493, 14.11.1965. (MO). - Veracruz, about 10 miles N of Teziutlán, leg.: D. S. \& H. B. Correll 28763, 06.01.1964. (TEX-LL, type of Rondeletia minor Lundell, isotype: MEXU).

Arachnothryx guerrerensis (Lorence) Borhidi, Acta Bot. Hung. 38: 140. 1993-94 (publ. 1995). Basionym: Rondeletia guerrerensis Lorence, Novon 1: 143. (1991).

Specimen examined: Mexico: Guerrero, Distr. Galeano, Carrizo, pié de la Cuesta, alt. 1750 m, leg.: G. B. Hinton 14738, 28.10.1939. (MO, paratype).

Arachnothryx hameliifolia (Dwyer et Hayden) Borhidi, Acta Bot. Hung. 28: 70. (1982). = Rondeletia hameliifolia Dwyer et Hayden, Ann. Mo. Bot. Gard. 54: 144. (1967).

Arachnothryx heteranthera (Brandegee) Borhidi, Acta Bot. Hung. 28: 69. (1982). Basionym: Rondeletia heteranthera Brandegee, Univ. Calif. Publ. Bot. 4: 387. (1913).

Specimens examined: Mexico: Hidalgo, 105 hwy entre Pachuca and Tampico, 18-23 miles al S de Huejutla, coord.: $20^{\circ} 59^{\prime} \mathrm{N}, 98^{\circ} 31^{\prime} \mathrm{W}$, alt. $900 \mathrm{~m}$, leg.: T. B. Croat \& D. P. Hannon 66003, 28.02.1987. (MO). - Hidalgo, km 332 of Mexico City-Laredo hwy, above Chapulhuacán, alt. 3800 ft, leg.: C. L. Lundell 12234. 13.07.1943. (MO, TEX-LL, type of Rondeletia falciformis Lundell). - Hidalgo, montane stream N of Chapulhuacán, leg.: O. M. Clark 01.06.1935. (MO). - Oaxaca, alrededores de Nueva Santa Flora, 11 km ESE de San Felipe Uzila, coord.: $17^{\circ} 54^{\prime} \mathrm{N}, 96^{\circ} 26^{\prime} \mathrm{W}$, leg.: G. I. Manriquez 3659, 26.07.1992. (MO). Oaxaca, Mpio. San Felipe Juzila, Nueva Santa Flora, coord.: $17^{\circ} 55^{\prime}$ N, $96^{\circ} 26^{\prime}$ W, leg.: G. I. Manriquez et al. 3763, 20.12.1992. (MO). - Veracruz, Banos de Carrizal, leg.: C. A. Purpus 
6184, 08.1912. (MO, isotype). - Veracruz, Mpio. Yecuatla, El Jaya, alt. 1220 m, leg.: F. Ventura A. 5156. (MO).

Arachnothryx izabalensis (Standl. et Steyerm.) Borhidi, Acta Bot. Hung. 33: 302. (1987). Basionym: Rondeletia izabalensis Standl. et Steyerm., Publ. Field Mus. Nat. Hist., Bot. Ser., 22: 286. (1940).

Arachnothryx jurgensenii (Hemsl.) Borhidi, Acta Bot. Hung. 28: 70. (1982). Basionym: Rondeletia jurgensenii Hemsl., Diag. Pl. Nov. Mexic., p. 29. (1879).

Specimens examined: Honduras: Cordillera Merendón, El Moral, alt. 1600 m, leg.: A. Molina R. 23285, 27.08.1968. (MO). - Dept. F. Morazán, Epaterique, riverbank thicket, alt. 1600 m, leg.: A. Molina R. \& A. R. Molina 25847, 26097, 26107, 11.08.1971. (MO). Mexico: Aguazatca-Silo, Mina Gro, leg.: G. B. Hinton, 10493, 13.07.1930. (MO). - Cumbre de Tijuquico, Temascaltepec, leg.: G. B. Hinton 7016, 22.11.1934. (MO). - Distr. Temascaltepec, Nanchititla, Barranca, leg.: G. B. Hinton 4517. (MO) (f. pubescens). - Las Mesas, Temascaltepec, leg.: G. B. Hinton 6394, 05.05.1934. (MO). - Nayarit, Cerro de San Juan, W of Tepic, alt. 1000 m, leg.: Y. Mexía 727, 19.09.1926. (MO, type). - Nayarit, Mpio. Jalisco, 3.5 km sobre la terracería a El Tacote al SW de Jalisco, carretera Tepic-Puerto Vallarta, coord.: $21^{\circ} 27^{\prime} \mathrm{N}, 105^{\circ} 56^{\prime} \mathrm{W}$, alt. $1180 \mathrm{~m}$, leg.: O. Téllez 12782, 01.08.1990. (MO) (with fl.!). - Oaxaca, Dept. Míahuatlán, al km 622 camino a Piedra Larga, Míahuatlán, leg.: R. T. Cedillo \& R. C. Torres 1821, 24.09.1972. (MO) (with fr.!). - Oaxaca, Distr. de Putla, a $1 \mathrm{~km}$ al N de la desviación a Iuxtalahuaca, viniendo de Tlaxiago, alt. $1620 \mathrm{~m}$, leg.: R. C. Torres \& H. Hernandez 3357, 30.07.1983. (MO). - Oaxaca, Distr. Iuxtlahuaca, $10 \mathrm{~km}$ al S de Copala, carretera San Augustin a Tenango-Putla, alt. 2720 m, leg.: R. C. Torres \& R. T. Cedillo 595, 1982. (MO). - Oaxaca, Sierra de San Pedro Nolasco, leg.: Jurgensen 248. (MO).

Arachnothryx kirkbridei (Dwyer) Borhidi, Acta Bot. Hung. 28: 70. (1982). Basionym: Rondeletia kirkbridei Dwyer, Ann. Mo. Bot. Gard. 67: 469. (1980). = Arachnothryx bertieroides (Standl.) Borhidi.

Arachnothryx laniflora (Benth.) Planch., Fl. Serres Jard. 5: tab. 442. (1849). Basionym: Rondeletia laniflora Benth., Pl. Hartweg., p. 85. (1841).

Specimens examined: El Salvador: Cerro Verde, alt. 1500 m, leg.: A. F. Clewell 3675, 18.02.1973. (MO). - Dept. Ahuachapán, Cerro Grande de Apanéa, leg.: J. D. Dwyer 77,

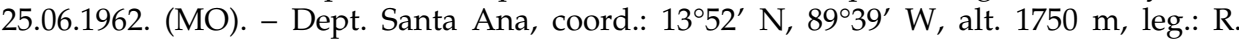
Villacorta \& S. Martinez 363, 11.08.1983. (MO). - Dept. Santa Ana, ladera N del Volcán Santa Ana, coord.: $13^{\circ} 41^{\prime}$ N, 89 53' W, alt. 1780 m, leg.: M. L. Reyna \& R. Villacorta 1445, 09.06.1989. (MO). - Dept. Santa Ana, Volcán de Santa Ana, Ojo de Agua, leg.: J. Jurado, 31.03.1987. (MO). - Distr. Santa Ana, Cerro Verde National Park, coord.: $13^{\circ} 29^{\prime} \mathrm{N}, 88^{\circ} 32^{\prime} \mathrm{W}$, alt. 2300 m, leg.: K. Sidwell et al. 438, 23.01.1998. (MO). - Mpio. de Chalchuapa, entre El Olimpo y la cima del Cerro El Ojo de Agua de la Virgen, $21 \mathrm{~km}$ al SO de la ciudad de Santa Ana, alt. 1930 m, leg.: J. L. Linares \& C. A. Martinez 2367, 26.03.1995. (MO). Mexico: Chiapas, Motozintla, alt. 2176 m, leg.: E. Matuda 5509, 04.1945. (MO, type of Rondeletia albida Lundell). - Chiapas, along hwy between Huixtla and Motozintla de Mendoza, in vi- 
cinity of El Rosario, alt. 1850 m, leg.: T. B. Croat 47232, 10.02.1972. (MO). - Chiapas, along the dirty road to Siltepec, past Ejído, Benito Juárez, ca $12 \mathrm{~km}$ from the turnoff from Mexican Hwy 190, S of Motozintla, coord.: $15^{\circ} 20^{\prime}$ N, $92^{\circ} 15^{\prime}$ W, alt. 2100 m, leg.: J. S. Miller \& J. K. Myers 2755, 09.05.1987. (MO). - Chiapas, Cerro de Boquerón, leg.: C. A. Purpus 7227, 06.1914. (MO). - Chiapas, Cerro Mozotal, $11 \mathrm{~km}$ from NW of junction of road to Motozintla along the road to El Porvenir and Siltepec, leg.: D. E. Breedlove 25881, 27.06.1972. (MO). Chiapas, Distr. Motozintla de Mendoza, Cerro Mozotal, $11 \mathrm{~km}$ NW of the junction on the road to Motozintla, along the road to El Porvenir and Siltepec, alt. 2100 m, leg.: D. E. Breedlove 55747, 23.11.1981. (MO, with fr.!). - Chiapas, Cerro Tres Picos, alt. 2100-2500 m, leg.: D. E. Breedlove 25383. (MO). - Chiapas, Letrero near Siltepec, alt. 2000 m, leg.: E. Matuda, 4328, 06.07.1941. (MO). - Chiapas, Llano Grande, $4 \mathrm{~km}$ al NE de Mpio. de Motozintla, alt. 2900 m, leg.: E. Ventura \& E. López 4378. (MO, with fl.!). - Chiapas, Mpio. Mapastepec, area natural de la Reserva El Triunfo, en el Cipresal, leg.: A Reyes García 1709, 02.03.1990. (MO). - Chiapas, Mpio. Mapastepec, area natural de la reserva El Triunfo, el Cipresal, alt. 1800 m, leg.: A. Reyes García 1714, 02.03.1990. (MO). - Chiapas, Mpio. Motozintla de Mendoza, $50 \mathrm{~km}$ NE of Huixtla, on road to Motozintla, alt. $1900 \mathrm{~m}$, leg.: D. E. Breedlove \& R. F. Thorne 30993, 28.12.1972. (MO). - Chiapas, Mpio. Motozintla de Mendoza, 8 miles W of junction with Motozintla-Iuxtka hwy, along the road to Motozal and Siltepec, alt. $6600 \mathrm{ft}$, coord.: $15^{\circ} 21^{\prime}$ N, $92^{\circ} 20^{\prime}$ W, leg.: D. Neil 5593, 02.04.1983. (MO). Chiapas, Mpio. Motozintla, terracería a $11 \mathrm{~km}$ al O de Buenos Aires, alt. $2000 \mathrm{~m}$, leg.: A. Mendez Ton 5785, 02.04.1983. (MEXU, MO). - Chiapas, Mpio. Siltepec, leach above Siltepec, on the road to Huixtla, alt. 2000-2400 m, leg.: D. E. Breedlove \& A. R. Smith 31922, 18.01.1973. (MO). - Chiapas, Mt. Ovando, leg.: E. Matuda 861, 05.04.1936. (MO). - Chiapas, Mt. Ovando, leg.: E. Matuda, 09.04.1937. (MO). - Chiapas, Mt. Ovando, leg.: E. Matuda S-91, 04.04.1938. (MO). - Chiapas, Mt. Pasitar, leg.: E. Matuda 1687, 03.08.1937. (MO, 2 spec.). Chiapas, track from Ejido to Boquerón, Cerro Boquerón, coord.: $15^{\circ} 15^{\prime} \mathrm{N}, 92^{\circ} 17^{\prime} \mathrm{W}$, alt. 2400 m, leg.: P. J. Stafford et al. 348, 09.02.1990. (MO). - Oaxaca, Dept. Miahuatlán, Mpio. de San Juan Coatlán, 42 km al SO de San Jerónimo Coatlán, brecha a Progresu, leg.: A. Campos V. 3156, 26.06.1990. (MO). - Oaxaca, Mpio. San Jerónimo, Dept. Miahuatlán, $30 \mathrm{~km}$ al SO de San Juan Coatlán, brecha a Piedra Larga, alt. 1500 m, coord.: $16^{\circ} 11^{\prime} \mathrm{N}, 96^{\circ} 58^{\prime} \mathrm{W}$, leg.: A. Campos V. 3294, 08.08.1990. (MO). - Veracruz, hills above and NE of Xico Viejo, $8 \mathrm{~km} \mathrm{NW}$ of Xico, leg.: M. Nee \& K. Taylor 29378, 05.02.1985. (MO). - Veracruz, Xico, Cerro Punta, Coscomat, leg.: E. Matuda 1503, 10.05.1937. (MO).

Arachnothryx leptodictya (B. L. Robins.) Borhidi, Acta Bot. Hung. 28: 68. (1982). Basionym: Rondeletia leptodictya B. L. Robins., Proc. Amer. Acad. Sci. 45: 402. (1910). = Arachnothryx leucophylla (Kunth) Planch.

Arachnothryx leucophylla (Kunth) Planch., Fl. Serres Jard. 5: tab. 442. (1849). Basionym: Rondeletia leucophylla Kunth in Humb., Bonpl. and Kunth, Nov. Gen. Spec. 3: 395, tab. 290. (1820).

Specimens examined: Mexico: Durango, steep side cańón of barranca del Rio Tamazula, between the settlements of Agua Caliente and La Bajada, alt. 700 m, leg.: D. E. Breedlove 24472, 19.03.1972. (MO). - Guerrero, en casa privada, leg.: D. H. Lorence 4553, 28.12.1984. (MO). - Guerrero, Temisco, lower barranca de la Guacamaya, border stream open, alt. 200 m, leg.: Y. Mexía 8886, 24.11.1937. (MO). - Jalisco, Las Mesitas, NW od San 
Sebastián, arroyo de las Casitas, Sierra Madre, alt. 1700 m, leg.: Y. Mexía 1880, 15.03.1927. (MO). - Jalisco, Mpio. Tonila, $21 \mathrm{~km}$ al SO de Atenquique, carrertera Tonila-Atenquique canón que da al rio Tuxpan, leg.: E. J. Lott 795, 20.11.1981. (MO). - Jalisco, Puerto Vallarta, leg.: Y. Mexía 1312, 17.12. 1926. (MO). - Jalisco, Puerto Vallarta, near to seashore, leg.: Y. Mexía 1136, 26.11.1926. (MO). - Michoacán, Coalcomán, alt. 1100 m, leg.: G. B. Hinton 1284, 30.12.1938. (MO). - Michoacán, Coalcomán, alt. 1100 m, leg.: G. B. Hinton 13638, 31.03.1939. (MO). - Nayarit, Mpio. Compostela La Cascada, 9 km NO de Mejías, coord.: $21^{\circ} 08^{\prime} \mathrm{N}$, $105^{\circ} 15^{\prime}$ W, alt. 470 m, leg.: G. Flores F899, 20.03.1989. (MO). - Nayarit, Mpio. El Nayarit, 19 $\mathrm{km}$ al NE de San Pedro Ixcatán, O a $2 \mathrm{~km}$ al NE de El Naranjo terrefería San Pedro

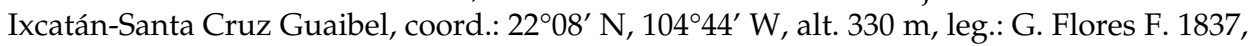
02.12.1989. (MO). - Nayarit, Mpio. Nayarit, Arroyo El Fraile al SE de la Mesa de Nayarit, coord.: $22^{\circ} 09^{\prime} \mathrm{N}, 104^{\circ} 33^{\prime} \mathrm{W}$, alt. 520 m, leg.: P. Tenorio L. \& G. Flores F. 16626, 23.10.1989. (MO). - Nayarit, Mpio. Santa Maria de Oro, $13 \mathrm{~km}$ carretera hacía la Laguna de Santa María del Oro, 2 km al N del poblado de Santa María de Oro, alt. 1140 m, leg.: C. Ismael Juan et al. 19153, 02.04.1994. (MO). - Nayarit, Mpio. Tepic Agua Milpa, $4 \mathrm{~km}$ antes de la presa, coord.: $21^{\circ} 40^{\prime} \mathrm{N}, 104^{\circ} 34^{\prime} \mathrm{W}$, alt. $170 \mathrm{~m}$, leg.: G. Flores F. 1863, 04.12.1989. (MO). - Nayarit, Mpio. Tepic, km 20-30 camino de terracería a Qurentelio-Cora-Jalcocotán en la serranía de San Juan, leg.: O. Téllez V. 11148, 14.01.1988. (MO). - Sinaloa, Capadero, Sierra Tacuichamona, alt. 3500 ft, leg.: B. Scott \& A. Gentry 5599. (MO).

Arachnothryx liebmannii (Standl.) Borhidi, Acta Bot. Hung. 33: 302. (1987). Basionym: Rondeletia liebmannii Standl., J. Wash. Acad. Sci. 17: 336. (1927). = Arachnothryx capitellata (Hemsl.) Borhidi.

Arachnothryx linguiformis (Hemsl.) Borhidi, Acta Bot. Hung. 28: 70. (1982). Basionym: Rondeletia linguiformis Hemsl., Diag. Pl. Nov. Mexic., p. 29. (1879).

Specimens examined: Guatemala: $1 \mathrm{~km}$ al O del Rio Blanco leg.: W. E. Harmon \& J. A. Fuentes 4882, 17.11.1970. (MO). - Dept. Alta Verapaz, 4 miles up road to Oxec, along road which course off the hwy, $6 \mathrm{~km}$ NE of Panzos, alt. $450 \mathrm{~m}$, leg.: T. B. Croat 41582, 20.07.1977. (MO). - Dept. Izabal, $15 \mathrm{~km} \mathrm{~W}$ of El Estor, leg.: W. E. Harmon \& J. D. Dwyer 4310, 09.04.1970. (MO). - Dept. Izabal, al Puerto Mendez, $19 \mathrm{~km}$ on Rio Dulce road, leg.: E. Contreras 10280, 10284, 14.09.1970. (MO). - Dept. Izabal, bordering road $4 \mathrm{~km}$ from the ruta atlantica, leg.: E. Contreras 10808, 22.05.1971. (MO). - Dept. Izabal, El Estor, leg.: E. Contreras 11443, 21.03.1972. (MO). - Dept. Izabal, en la Mina a $5 \mathrm{~km}$ O de El Estor, alt. $50 \mathrm{~m}$, leg.: E. M. Martinez S. 23287, 23298, 28.08.1988. (MO). - Dept. Izabal, entre El Benque y El Chorrero, carretera atlantico, leg.: R. Tún Ortíz 2446, 18.03.1972. (MO). - Dept. Izabal, hills above Exinbal, mining area, W of El Estor, leg.: W. E. Harmon \& J. D. Dwyer 4338, 09.04.1970. (MO). - Dept. Izabal, Mariscos bordering Lake Izabal, leg.: E. Contreras 7579, 10.02.1968. (MO). - Dept. Izabal, Mpio. El Estor, camino de Exmibal al E de Estor, coord.: $15^{\circ} 31^{\prime} \mathrm{N}, 89^{\circ} 23^{\prime} \mathrm{W}$, alt. $50 \mathrm{~m}$, leg.: P. Tenorio L. et al. 14565, 17.07.1988. (MO). - Dept. Izabal, on Petén-Guatemala road between Cienaga y Seja, leg.: E. Contreras 10873, 28.05.1971. (MO). - Dept. Izabal, slopes WSW above El Estor, alt. $210 \mathrm{~m}$, leg.: W. D. Stevens \& E. Martinez 25262, 30.08.1988. (MO). - Dept. Izabal, Los Amates, 18 km montana de Mico, leg.: W. E. Harmon 2568, 13.07.1980. (MO). - Dept. Izabal, Mpio. El Estor, La Cumbre, al O de Estor camino a la Finca Muca, coord.: $15^{\circ} 33^{\prime} \mathrm{N}, 89^{\circ} 24^{\prime} \mathrm{W}$, alt. $450 \mathrm{~m}$, leg.: P. Tenorio et al. 14498, 
17.07.1988. (MO). - Los Amates, Izabal, leg.: W. E. Harmon \& J. D. Dwyer 2865, 30.06.1970. (MO).

Arachnothryx longipetiolata (Lundell) Borhidi, Acta Bot. Hung. 35: 310. (1989). Basionym: Rondeletia longipetiolata Lundell, Wrightia 5: 325. (1976). = Arachnothryx buddleioides (Benth.) Planch.

Arachnothryx lundelliana (Standl.) Borhidi, Acta Bot. Hung. 33: 302. (1987). Basionym: Rondeletia lundelliana Standl., Contr. Univ. Mich. Herb. 4: 31. (1940). = Rogiera stenosiphon (Hemsl.) Borhidi.

Arachnothryx macrocalyx (Standl. et Steyerm.) Borhidi, Acta Bot. Hung. 33: 302. (1987). Basionym: Rondeletia macrocalyx Standl. et Steyerm., Publ. Field Mus. Nat. Hist., Bot. Ser. 23: 254. (1943). = Javorkaea macrocalyx (Standl. et Steyerm.) Borhidi, comb. nova hoc loco.

Arachnothryx manantlanensis (Lorence) Borhidi, Acta Bot. Hung. 38: 140. 1993-94 (publ. 1995) [as manantiensis]. Basionym: Rondeletia manantlanensis Lorence, Novon 1: 145. (1991).

Arachnothryx mexiae (Standl.) Borhidi, Acta Bot. Hung. 33: 302. (1987). Basionym: Rondeletia mexiae Standl., Publ. Field Mus. Nat. Hist., Bot. Ser. 22: 55. (1940). = Javorkaea scabra (Hemsl.) Borhidi.

Arachnothryx mexicana (Turcz.) Borhidi, comb. nova hoc loco. Basionym: Siphonandra mexicana Turcz., Bull. Soc. Imp. Naturalistes Moscou 21: 581. (1848). Synonym: Rondeletia mexicana (Turcz.) Standl., Publ. Field Mus. Nat. Hist., Bot. Ser. 22: 388. (1940).

Arachnothryx minor (Lundell) Borhidi, Acta Bot. Hung. 35: 310. (1989). Basionym: Rondeletia minor Lundell, Wrightia 5: 326. (1976). = Arachnothryx gracilispica (Standl.) Borhidi.

Arachnothryx monteverdensis (Lorence) Borhidi, Acta Bot. Hung. 38: 140. 1993-94 (publ. 1995). Basionym: Rondeletia monteverdensis Lorence, Novon 1: 147. (1991).

Specimens examined: Costa Rica: Prov. Guanacaste, Parque Rincón de la Vieja Hacienda Santa María, sendero que va del Mirador hasta los 1300 m, Falda del Volcán Santa María, alt. 1200 m, coord.: $10^{\circ} 48^{\prime}$ N, 85¹9’ W, leg.: G. Herrera 651, 08.08.1987. (MO). - Prov. Guanacaste, upper Rio Chiquito valley, $50 \mathrm{~km} \mathrm{~N}$ of Santa Elena, coord.: $10^{\circ} 25^{\prime} \mathrm{N}, 84^{\circ} 50^{\prime} \mathrm{W}$, alt. 1300 m, leg.: W. A. Haber 4379, 13.04.1986. (MO). - Puntarenas, Prov. Alajuela, settle- 
ment at Monteverde, alt. 1600-1700 m, leg.: J. \& C. Hutley 2394, 05.1975. (MO). Puntarenas, Reserva Biológica Monteverde, en Quebrada Veracruz, Finca Pablo Morales, coord.: $10^{\circ} 15^{\prime} \mathrm{N}, 84^{\circ} 18^{\prime} \mathrm{W}$, alt. 1600 m, leg.: E. Bello 1753, 11.01.1990. (MO). - San José, Parque Nacional Braulio Carrillo, sendero hacía la Quebrada Zurqui, alt. 1250 m, leg.: N. Zamora et al. 610, 30.06.1984. (MO).

Arachnothryx myriantha (Standl. et Steyerm.) Borhidi, Acta Bot. Hung. 33: 302. (1987). Basionym: Rondeletia myriantha Standl. et Steyerm., Publ. Field Mus. Nat. Hist., Bot. Ser. 22: 288. (1940).

Arachnothryx nebulosa (Standl.) Borhidi, Acta Bot. Hung. 33: 302. (1987). Basionym: Rondeletia nebulosa Standl., Trop. Woods 37: 32. (1934).

Specimens examined: Honduras: Aldea La Florida, $25 \mathrm{~km}$ al SE de la Ciudad de Marcala, alt. 1300 m, leg.: M. Martinez M. 129, 01.03.1981. (MO). - Atlantida, La Ceiba, El Recreo $35 \mathrm{~km}$ al O, leg.: N. Zamora 1740, 23.11.1991. (MO). - Cerro de la Tigra, Cerro Nebuloso, 20 km NE de Tegucigalpa, alt. 2100-2180 m, leg.: M. Erazo et al. 2168, 07.03.1957. (MO). - Comayagua, Cortigera de Montecillo, Montana de San Juanillo, trail between Cerro San Juanillo y La Danta, coord.: 14³0’ N, 8752’ W, alt. 1800-1900 m, leg.: G. Davidse \& T. Hawkins 34200, 05.05.1990. (MO). - Dept Morazán, Sierra Tigra near Tegucigalpa, leg.: H. F. Clewell 3802, 18.03.1973. (MO). - Dept. Colón, Trujillo, Montana Capiró, leg.: L. Viela 136, 30.12.1979. (MO). - Dept. Comayagua, San Juanillo, Siguatepeque, bosque nublado al lado del camino El Danto a Jesus de Otoro, cumbre de la montana Reserva Biológica de Montecillos, coord.: 14³0’ N, 8753 W', alt. 2020 m, leg.: P. House 8858, 02.03.1991. (MO). Dept. de Francisco Morazán, Tegucigalpa, Cerro La Tigra, alt. 2300 m, leg.: O. Martinez 110, 06.04.1980. (MO). - Dept. Francisco Morazán, Parque Nacional La Tigra, $21 \mathrm{~km}$ al N de Tegucigalpa, leg.: O. Téllez 8596, 04.06.1984. (MO, with fr.!). - Dept. Francisco Morazán, Parque Nacional La Tigra, a $25 \mathrm{~km}$ al NE de Tegucigalpa, coord.: $14^{\circ} 12^{\prime} \mathrm{N}, 87^{\circ} 07^{\prime} \mathrm{W}$, alt. 1850-2125 m, leg.: T. B. Croat \& D. P. Hannon 63991, 01.02.1987. (MO). - El Hatillo, alt. 1800 m, leg.: E. Vargas N. \& F. A. Barkley 01.08.1975. (MO). - La Paz, Las Trancas $5 \mathrm{~km}$ to the NW of Güajiquiro, coord.: $14^{\circ} 08^{\prime} \mathrm{N}, 87^{\circ} 52^{\prime} \mathrm{W}$, alt. 2000-2100 m, leg.: R. L. Liesner 26455, 22.05.1993. (MO). - Montana La Tigra, bosque de nubes, Tegucigalpa, alt. $1800 \mathrm{~m}$, leg.: C. Galeano 128, 29.02.1980. (MO, with fl.!). - Siguatepeque, Dept. Olancho, La Chorrera, coord.: $15^{\circ} 00^{\prime} \mathrm{N}, 85^{\circ} 56^{\prime} \mathrm{W}$, alt. $1140 \mathrm{~m}$, ambos lados del Rio Lara, alrededor de la Chorrera, $15 \mathrm{~km}$ al NE de Catacamas, leg.: M. Dario 121, 30.05.1992. (MO). - Zona de amortiguamiento del Parque Nacional La Tigra, $30 \mathrm{~km}$ NE de Tegucigalpa, alt. $1000 \mathrm{~m}$, leg.: R. Perdomo 294, 18.04.1982. (MO). nicaragua: Matagalpa, slopes of Cerro Picachonear the border, Dept. Jinotega, coord.: 1300' N, 8551' W, alt. 1200-1540 m, leg.: G. Davidse, A. Grijalva \& M. Sousa 30347, 23.05.1987. (MO).

Arachnothryx nicaraguensis (Oerst.) Borhidi, Acta Bot. Hung. 35: 310. (1989). Basionym: Rondeletia nicaraguensis Oerst., Vidensk. Meddel. Dansk Naturhist. Foren., Kjöbenhavn, 1852: 43. (1853). = Rogiera nicaraguensis (Oerst.) Borhidi, comb. nova hoc loco. 
Arachnothryx nitida (Hemsl.) Borhidi, Acta Bot. Hung. 28: 69. (1982). Basionym: Rondeletia nitida Hemsl., Diag. Pl. Nov. Mexic., p. 29. (1879).

Specimens examined: Mexico: Chiapas, $5 \mathrm{~km}$ SE of Jitotol along road to Bochil, Mpio. Jitotol, alt. 1600 m, leg.: D. E. Breedlove 23313, 19.12.1971. (MO). - Chiapas, about $12 \mathrm{~km} \mathrm{~N}$ of Jitotol, along the sideroad to an oilwell, Mpio. Jitotol, alt. $2000 \mathrm{~m}$, leg.: D. E. Breedlove \& R. F. Thorne 21482, 28.10.1972. (MO). - Chiapas, along logging road from Las Margaritas to Campo Alegre, Mpio. La Independencia, alt. 2300 m, leg.: D. E. Breedlove 33542, 18.02.1943. (MO). - Chiapas, along the logging road from Las Margaritas to Campo Alegre, Mpio La Independencia, alt. 2300 m, leg.: D. E. Breedlove \& F. Almeda 47858, 27.11.1980. (MO). Oaxaca, $25 \mathrm{~km}$ al $\mathrm{N}$ de Humo Chico, carretera a Oaxaca-Tuxtepec, Mpio. de Ixtlán, leg.: R. C. Torres \& R. T. Cedillo 1437, 27.09.1982. (MO). - Oaxaca, Distr. Ixtlán, para la Cascada Cara N del Cerro Pelón, carretera Oaxaca-Tuxtepec, alt. 2300 m, leg.: A. García M., D. H. Lorence \& C. Alain 1847, 04.08.1985. (MO, with fr.!). - Oaxaca, along hwy 175, between Valle Nacional and Oaxaca, km 98 NNE of Hualtianguis, 37 miles NNE of Ixtlán de Juárez, alt. 2200 m, coord.: $17^{\circ} 37^{\prime}$ N, $96^{\circ} 22^{\prime}$ W, leg.: T. B. Croat \& D. P. Hannon 65586, 22.02.1987. (MO). - Oaxaca, Distr. Ixtlán de Juárez, carretera Valle Nacional a Ixtlán, al SO de Vista Hermosa, alt. 2040 m, leg.: R. T. Cedillo, D. H. Lorence \& A. García 921, 03.08.1981. (MO). Oaxaca, Distr. de Ixtlán, Mpio de Yolox, 9 km N de la desviación de Yolox, alt. 2400 m, leg.: G. J. Martinez 487, 07.04.1987. (MO). - Oaxaca, Distr. de Ixtlán, ruta 175 Oaxaca a Tuxtepec, 9 km N de la desviación de Yolox. leg.: D. H. Lorence \& G. Martin 3221, 07.04.1981. (MO). Oaxaca, Distr. Ixtlán, Mpio. Comaltepec, just of hwy 175, Ixtlán de Juárez to Valle Nacional, caribbean slope, coord.: $17^{\circ} 35^{\prime} 35^{\prime \prime}$ N, 96 $96^{\prime} 26^{\prime \prime} \mathrm{W}$, alt. 2250 m, leg.: P. \& A. Boyle 527. (MO).

Arachnothryx oaxacana (Standl.) Borhidi, Acta Bot. Hung. 33: 302. (1987). Basionym: Rondeletia oaxacana Standl., Field Mus. Nat. Hist., Bot. Ser. 11: 255. (1936). = Arachnothryx mexicana (Turcz.) Borhidi.

Arachnothryx ovandensis (Lundell) Borhidi, Acta Bot. Hung. 35: 310. (1989). Basionym: Rondeletia ovandensis Lundell, Wrightia 5: 325. (1976).

Arachnothryx pansamalana (Standl.) Borhidi, Acta Bot. Hung. 28: 69. (1982). Basionym: Rondeletia pansamalana Standl., N. Amer. Fl. 32: 58. (1918).

Arachnothryx phyllocephala (Standl. et L. O. Williams) Borhidi, Acta Bot. Hung. 33: 302. (1987). Basionym: Rondeletia phyllocephala Standl. et L. O. Williams, Ceiba 3: 63. (1952). = Arachnothryx thiemei (Donn.Sm.) Borhidi.

Arachnothryx platysepala (Standl.) Borhidi, Acta Bot. Hung. 28: 70. (1982). Basionym: Rondeletia platysepala Standl., Ann. Mo. Bot. Gard. 27: 343. (1940). = Arachnothryx bertieroides (Standl.) Borhidi. 
Arachnothryx polycephala (Standl.) Borhidi, Acta Bot. Hung. 35: 310. (1989). Basionym: Rondeletia polycephala Standl., J. Wash. Acad. Sci. 17: 337. (1927). = Arachnothryx mexicana (Turcz.) Borhidi.

Arachnothryx povedae (Lorence) Borhidi, Acta Bot. Hung. 38: 140. 1993-94 (publ. 1995). Basionym: Rondeletia povedae Lorence in W. C. Burger \& C. M. Taylor, Fieldiana, Bot., nov. ser., 33: 299. (1993).

Specimens examined: Costa Rica: San José, Santa Rosa de Puriscal, leg.: L. J. Poveda 441, 17.01.1973. (MO, isotype). - San José, Reserva Biológica Carrara, sector Rio Carrara, coord.: $9^{\circ} 45^{\prime} \mathrm{N}, 84^{\circ} 33^{\prime} 50^{\prime \prime} \mathrm{W}$, alt. $150 \mathrm{~m}$, leg.: R. Zuniga 412, 05.01.1991. (MO). - Puntarenas, Reserva Biológica Carrara, sendero Quebrada Bonita a Bijagual, Lomas Pisote, coord.: $9^{\circ} 47^{\prime} 10^{\prime \prime} \mathrm{N}, 84^{\circ} 35^{\prime} 10^{\prime \prime} \mathrm{W}$, alt. $300 \mathrm{~m}$, leg.: R. Zuniga \& Q. Jimenez 15, 08.12.1989. (MO).

Arachnothryx pringlei (Lorence) Borhidi, Acta Bot. Hung. 40: 16. 1996-97 (1999). Basionym: Rondeletia pringlei Lorence, Novon 7: 54-55. (1997).

Specimens examined: Mexico: Hidalgo, along hwy 105 between Pachuca and Tampico, along road to San Cristóbal, coord.: $20^{\circ} 58^{\prime} \mathrm{N}, 98^{\circ} 38^{\prime} \mathrm{W}$, alt. $1550 \mathrm{~m}$, leg.: T. B. Croat \& D. P. Hannon 65888, 28.02.1987. (MO, with fl.!). - Hidalgo, Mpio Tenango de Doria, camino de Tenango de Doria a El Cirillo, penascos al $6 \mathrm{~km}$ de Tenango, alt. 1750-1800 m, leg.: D. H. Lorence \& R. M. Hernandez 4903, 09.11.1983. (MO). - Hidalgo, Tlajuelonpan, 12 km al S de Xacualtipán, Mpio. Xacualtipán, alt. 1900 m, leg.: R. Hernandez M. \& P. Tenorio L. 7091, 04.03.1982. (MO). - Veracruz, hills near Orizaba, alt. 5000 ft, leg.: C. G. Pringle 6135, 09.02.1895. (MEXU, MO, isotype). - Veracruz, Mpio. La Banderilla, Bileteas cerca de Banderilla, alt. 1600 m, leg.: R. Hernandez M. \& G. Dorantes L. 1766, 11.01.1973. (MO). Veracruz, Mpio. Xico, Sancheria de Cuatitlán, coord.: $19^{\circ} 29^{\prime}$ N, $97^{\circ} 04^{\prime}$ W, alt. 1820 m, leg.: J. J. I. Calzada 1747, 11.01.1984. (MO).

Arachnothryx purpurea (Lorence) Borhidi, Acta Bot. Hung. 38: 140. 1993-94. (publ. 1995). Basionym: Rondeletia purpurea Lorence, Novon 1: 148. (1991).

Arachnothryx pyramidalis (Lundell) Borhidi, Acta Bot. Hung. 35: 310. (1989). Basionym: Rondeletia pyramidalis Lundell, Wrightia 5: 327. (1976).

Specimens examined: Mexico: Chiapas, Jaltenango, El Triunfo Reserve, trail from El Triunfo Camp to Finca Prusia, ca 1 km from camp, coord.: $15^{\circ} 39^{\prime}$ N, $92^{\circ} 48^{\prime}$ W, alt. ca $2000 \mathrm{~m}$, leg.: R. J. Hampshire et al. 800, 03.03.1990. (MO). - Chiapas, San Luis near Siltepec, alt. 1300 m, leg.: E. Matuda 5292, 24.01.1945. (MEXU, MO, isotype). - Chiapas, Mpio. Siltepec, ridge above Siltepec, along the road to Huixtla, alt. 2000-2400 m, leg.: D. E. Breedlove \& A. R. Smith 31923, 18.01.1973. (MO). - Chiapas, Mpio. Siltepec, ridge above Siltepec, on the road to Ixtlá, alt. 2000-2400 m, leg.: D. E. Breedlove 40411. (MO). - Chiapas, Mpio. Villa Corzo, Cerro Tres Picos near Cerro Bola, along a logging road SW of Colonia Agrónomos Mexicanos, alt. 1500-1800 m, leg.: D. E. Breedlove 24982, 04.05.1972. (MO). - Oaxaca, along 
hwy 175, Sierra de Juárez, between Tuxtepec and Oaxaca, km 166, 13.6 miles S of bridge del Valle Nacional, alt. 1400 m, leg.: T. B. Croat 47957, 19.02.1979. (MO). - Oaxaca, km 124 along hwy 175 ca $50 \mathrm{~km} \mathrm{~S}$ of Tuxtepec, alt. 1500-2500 m, leg.: J. \& R. Conrad 3222, 29.08.1974. (MO).

Arachnothryx rekoi (Standl.) Borhidi, Acta Bot. Hung. 28: 69. (1982). Basionym: Rondeletia rekoi Standl., Proc. Biol. Soc. Wash. 8: 126. (1918).

Specimens examined: Mexico: Oaxaca, Las Pilas, (Cerro Espino), alt. 400 m, leg.: B. P. Reko 3490, 10.10.1917. (MO, type). - Oaxaca, Mpio. Numa Hidalgo, terracería a Tultepec 1 $\mathrm{km}$ al E de la carretera Pochutla-Oaxaca, $16 \mathrm{~km}$ N de Pochutla, alt. $320 \mathrm{~m}$, leg.: S. D. Koch, E. A. Fryxell \& T. Wendt 79493. (MO).

Arachnothryx ricoi (Lorence) Borhidi, Acta Bot. Hung. 38: 140. 1993-94 (publ. 1995). Basionym: Rondeletia ricoi Lorence, Novon 1: 152. (1991).

Arachnothryx rubens (L. O. Williams) Borhidi, Acta Bot. Hung. 35: 311. (1989). Basionym: Rondeletia rubens L. O. Williams, Phytologia 26: 128. (1973).

Arachnothryx rufescens (B. L. Robins.) Borhidi, Acta Bot. Hung. 28: 69. (1982). Basionym: Rondeletia rufescens B. L. Robins., Proc. Amer. Acad. Arts 45: 402. (1910). It is a good distinct species, not identical with Rondeletia gracilis Hemsl. As it is stated by Lorence in the Nomenclator (Lorence 1999).

Specimens examined: Guatemala: Dept. Alta Verapaz, Cobán, alt. 4800 ft, leg.: H. von Türckheim 582, 03.1881. (MO). - Dept. Alta Verapaz, Cobán in Walde, alt. 1600 m, leg.: H.von Türckheim, 03.1908. (MO). - Dept. Alta Verapaz, Tactic, alt. 1800 m, leg.: H. von Türckheim 8401, 03.1903. (MO, phototype). - Dept. Baja Verapaz, 4 km S de Puruljá, camino Guatemala-Cobán, cerca del Biotopo, alt. 1660 m, leg.: E. M. Martinez S. \& O. Téllez 13140, 19.06.1985. (MO). - Dept. Baja Verapaz, Unión Barrios, leg.: E. Contreras 10989, 20.02.1972. (MO). - Dept. Baja Verapaz, Unión Barrios, Usalama-Cobán road, S of km 147 of the hill, leg.: C. L. Lundell \& E. Contreras 18926, 05.02.1975. (MO). Honduras: Dept. de Ocotepeque, camino de Yaruchel Abelín Gualcho, alt. 1500-2000 m, leg.: C. Nelson et al. 3946, 2-15.04.1977. (MO). - Dept. Ocotepeque, $35 \mathrm{~km}$ al NE de Nuevo Ocotepeque, camino a San Pedro Sula, leg.: E. M. Martinez \& O. Téllez 12950, 12.06.1985. (MO). - Dept. Ocotepeque, Belén Gualcho, just outside the town on road to Corquín, leg.: S. Blackmoore \& M. Chorley 3857, 11.05.1987. (MO). - Dept. Ocotepeque, Belén, Gualchú, leg.: S. Blackmoore \& M. Chorley 3876, 12.05.1987. (MO). - Dept. Ocotepeque, Cordillera Merendón above El Portillo, $20 \mathrm{~km}$ al E de Nueva Ocotepeque, alt. $2000 \mathrm{~m}$, leg.: A. Molina R. et al. 31324, 17.01.1976. (MO). 
Arachnothryx rzedowskii (Lorence) Borhidi, Acta Bot. Hung. 38: 140. 1993-94 (publ. 1995). Basionym: Rondeletia rzedowskii Lorence, Novon 1: 154. (1991).

Arachnothryx salicifolia (Dwyer et Hayden) Borhidi, Acta Bot. Hung. 28: 70. (1982). Basionym: Rondeletia salicifolia Dwyer et Hayden, Phytologia 15: 58. (1967). = Arachnothryx bertieroides (Standl.) Borhidi.

Arachnothryx scoti (Lorence) Borhidi, Acta Bot. Hung. 38: 140. 1993-94 (publ. 1995). Basionym: Rondeletia scoti Lorence, Novon 1: 155. (1991).

Specimens examined: Mexico: Oaxaca, La Glorieta, Rio Grande (N slope), leg.: T. McDougall s. n., 12.03.1953. (isotype: MO, holotype: MEXU!).

Arachnothryx secunda (Standl.) Borhidi, Acta Bot. Hung. 28: 70. (1982). Basionym: Rondeletia secunda Standl., Contr. U.S. Natl. Herb. 18: 141. (1916).

Specimens examined: Panama: Prov. Colón, boat bank just W of Porto Belo, leg.: W. G. D'Arcy 4079, 02.04.1970. (MO, type of Rondeletia d'arcii Dwyer). - San Blas, Comarca de San Blas, bajando por el Rio Playón Chico desde Nebat Dummat, coord.: $9^{\circ} 15^{\prime} \mathrm{N}, 78^{\circ} 15^{\prime} \mathrm{W}$, alt. 40-50 m, leg.: H. Herrera 1689, 13.07.1990. (MO). - Playón Chico and vicinity of San Blas, Pincanti $6 \mathrm{~km}$ bush at bottom of the hill, leg.: F. Stier 183, 07.07.1975. (MO). - Prov. San Blas, forest above beach E of Puerto Obaldia, leg.: T. B. Croat 16919, 19.08.1971. (MO). - Prov. San Blas, hill E of Puerto Obaldia, leg.: W. G. D'Arcy 14249, 19.01.1981. (MO). - Prov. San Blas, headwaters of Rio Caudí, Camp Diablo, coord.: $82^{\circ} 02^{\prime} \mathrm{N}, 87^{\circ} 08^{\prime} \mathrm{W}$, alt. $273 \mathrm{ft}$, leg.: J. A. Duke, A. G. Robyns \& S. E. Verhoek 3657, 19.12.1967. (MO).

Arachnothryx secundiflora (B. L. Robins.) Borhidi, Acta Bot. Hung. 28: 69. (1982). Basionym: Rondeletia secundiflora B. L. Robins., Proc. Amer. Acad. Arts 45: 403. (1910).

Specimens examined: Guatemala: Dept. Texcuintla, $8 \mathrm{~km} \mathrm{~S}$ of Padín, leg.:W. E. Harmón \& J. D. Dwyer 2955, 08.07.1970. (MO). Honduras: Siguatepeque, Dept. Cobán, la Meseta, S slope of Cerro Azul, N above Quebrada Grande, $12 \mathrm{~km}$ NE of Florida, coord.: $15^{\circ} 06^{\prime} \mathrm{N}, 88^{\circ} 55^{\prime} \mathrm{W}$, alt. $1680 \mathrm{~m}$, leg.: T. Hawkins \& D. Mejía 202, 09.02.1992. (MO). Mexico: Chiapas, along dirt road between San Fernando and Moravillas near Lago Mal Paso, coord.: $15^{\circ} 53^{\prime} \mathrm{N}, 93^{\circ} 16^{\prime} \mathrm{W}$, alt. 850-950 m, leg.: T. B. Croat \& D. P. Shannon 65026, 15.02.1987. (MO). - Chiapas, Mpio. Cacaohuatán, Unión Roja, alt. 400 m, leg.: F. Ventura \& E. López 1466, 04.10.1984. (MO). - Chiapas, Mpio. Huixtla, forest $6 \mathrm{~km} \mathrm{~N}$ of Huixtla road to Motozintla, alt. 200 m, leg.: D. E. Breedlove \& F. Almeda 17683, 22.11.1980. (MO). - Chiapas, Mpio. Ocosingo, El Naha, al $15 \mathrm{~N}$ de Monte Líbano, camino a Chancala, coord.: $17^{\circ} 56^{\prime} \mathrm{N}, 96^{\circ} 27^{\prime} \mathrm{W}$, alt. 860 m, leg.: E. Martinez S. 18009, 04.1986. (MO). - Chiapas, Mpio. Ocosingo, forest 70 km SW of Palenque, on road to Ocosingo, along the Yoel Jucum, alt. $550 \mathrm{~m}$, leg.: D. E. Breedlove \& F. Almeda 48365, 04.12.1980. (MO). - Chiapas, Mpio. Ocosocoautla, 26-28 km N of Ocosocoautla along road to Mal Paso, alt. 700 m, leg.: D. E. Breedlove 22468, 15.11.1971. 
(MO). - Ibid., leg.: D. E. Breedlove 38190, 06.10.1974. (MO). - Chiapas, Mpio. San Felipe de Uzila, Trayecto Nuevo Santa Flora a arroyo Iguana, alt. 500-800 m, leg.: G. I. Manriquez 3681, 27.09.1992. (MO). - Chiapas, Mpio. Tila, Chebupay, alt. 1000 m, leg.: A. Mendez Ton 5237, 10.12.1982. (MEXU, MO). - Oaxaca, Distr. Juquila, 2 km al NE del Puente El Alacrán, camino a la Finca Pacífico, leg.: R. T. Cedillo \& R. C. Torres 1530, 25.06.1982. (MO). - Oaxaca, Mpio. Santa María Chimalapa, cabecera del arroyo Sardina, ca $1 \mathrm{~km} \mathrm{~S}$ a la vereda de Carrizal, leg.: A. Hernandez G. 300, 06.08.1984. (MO). - Oaxaca, Mpio. Santa María Chimalapa, Rio Milagro cerca de la desembocadura del arroyo Sangre, leg.: C. Gonzalez 1, 31.08.1985. (MO, with fl.!). - Oaxaca, Valle Nacional, roadside, 8.5 miles NE, leg.: D. Turm et al. 250, 08.10.1979. (MO). - Tabasco, vicinity of Teapa, along road between Teapa and Tacotalpa, 3.1 miles E of Teapa along stream and limestone cliffs, coord.: $17^{\circ} 33^{\prime} \mathrm{N}, 92^{\circ} 59^{\prime} \mathrm{W}$, alt. 150 m, leg.: T. B. Croat \& D. P. Shannon 65326, 1987. (MO). - Veracruz, km 7 camino Cedillo-La Esquadra, por el rio Solosuchil, Hidalgotitlán, leg.: B. Dorantes 3548, 28.09. 1974. (MO). - Veracruz, Laguna Encantada, 6 km al N de San Andrés Tuxtla, leg.: J. I. Calzada 958, 16.01.1973. (MO). - Veracruz, Loma Larga, $\mathrm{N}$ de la Laguna de Catemaco, alt. $290 \mathrm{~m}$, leg.: M. Sousa 2808, 17.09.1966. (MEXU, MO). - Veracruz, Mpio. Hidalgotitlán, Rio Solosuchil, entre Cedillo y La Esquadra, leg.: B. Vazquez 1488, 12.07.1974. (MO). - Veracruz, Mpio. Santiago Tuxtla, Lagura crater, Pollinapa, a km 18 en la carretera San Andrés Tuxtla, leg.: R. T. Cedillo 406, 22.10.1974. (MO). - Veracruz, Totonicapán, 3 km al N de Catemaco, leg.: R. T. Cedillo 2512, 14.09.1983. (MO).

Arachnothryx septicidalis (B. L. Robins.) Borhidi, Acta Bot. Hung. 28: 69. (1982). Basionym: Rondeletia septicidalis B. L. Robins., Proc. Amer. Acad. Arts 45: 403. (1910).

Specimens examined: Guatemala: Dept. San Marcos, 1 mile above Africa, ca 3.3 miles above Finca Armenia, above San Rafael, alt. 1600 m, leg.: T. B. Croat 40938, 13.07.1977. (MO). - San Marcos, Finca Armenia, San Rafael de Cuesta, leg.: J. D. Dwyer 14446, 07.07.1977. (MO). - San Marcos, Finca Armenia, San Rafael, pié de la Cuesta a Carrizal, leg.: J. D. Dwyer 15281, 09.08.1980. (MO). - Chiapas, Volcano Tacaná, al norte del volcán, alt. 2100 m, leg.: E. Matuda 2965, 02.04.1939. (MO).

Arachnothryx silvicola (L. O. Williams) Borhidi, Acta Bot. Hung. 35: 311. (1989). Basionym: Rondeletia silvicola L. O. Williams, Phytologia 26: 128 (1973).

Arachnothryx skutchii (Standl. et Steyerm.) Borhidi, Acta Bot. Hung. 33: 302. (1987). Basionym: Rondeletia skutchii Standl. et Steyerm., Publ. Field Mus. Nat. Hist., Bot. Ser. 22: 289. (1940).

Arachnothryx stachyoidea (Donn.Sm.) Borhidi, Acta Bot. Hung. 28: 69. (1982). Basionym: Rondeletia stachyoidea Donn.Sm., Bot. Gaz. 42: 298. (1906).

Specimens examined: belize: Distr. Toledo, Grimmi Cat, Salamanca, $40 \mathrm{~m}$, leg.: C. Whitefoord 1858, 25.15.1979. (MO). - Distr. Toledo, Blue Creek, leg.: C. Whitefoord 3177, 10.06.1981. (MO). - Distr. Toledo, NE side of Blue Creek upstream from Mayan Indian Vil- 
lage of Blue Creek, alt. ca $250 \mathrm{ft}$, coord.: $16^{\circ} 14^{\prime} \mathrm{N}, 8^{\circ} 00^{\prime} \mathrm{W}$, leg.: S. R. Hill 20265, 20.03.1989. (MO). - Distr. Toledo, trail to Esperanza beginning 1 mile N of Colombia Forest Station, leg.: J. D. Dwyer 11135, 12.06.1973. (MO). - Distr. Toledo, San José, 6.7 miles N of Colombia Forest Station, leg.: J. D. Dwyer 11178, 13.06.1973. (MO). - Distr. Toledo, 1.5 miles of Mayan Village of San José, on road to Colombia Forest Station, leg.: A. Gentry 8116, 12.07.1973. (MO). - Distr. Toledo, vicinity of San José, Mayan Indian Village, 6.7 miles N of Colombia Forest Station, leg.: T. B. Croat 24368, 13.06.1973. (MO). british honduras: Columbia Forest Station, leg.: J. D. Dwyer 9844, 24.06.1972. (MO). - river bank in open place at Rio Grande, alt. $400 \mathrm{ft}$, leg.: W. A. Schipp 1162, 21.04.1933. (MO). guatemala: Dept. El Petén, foresta baja Santa Elena orillando el camino para Coctún, km 25, leg.: R. Tún Ortíz 1077. (MO). - Dept. El Petén, La Cumbre, Sapolul in zapotal on hillside SWW, leg.: C. L. Lundell \& E. Contreras 20788, 12.04.1977. (MO). - El Petén, San Luis, ca $50 \mathrm{~km}$ of road on the ringstream rain forest. leg.: C. L. Lundell 16363, 11.07.1959. (MO). - Dept. Izabal, El Estor, in high forest, leg.: E. Contreras 11344, 14.03.1972. (MO). - Izabal, road Las Escobas, towards Cerro San Gil, alt.

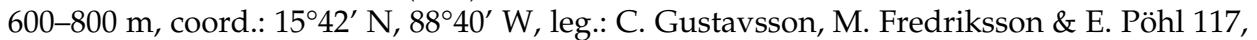
21.02.1993. (MO). - Ibid., alt. 300-500 m, leg.: C. Gustavsson, M Fredriksson \& E. Pöhl 123, 21.02.1993. (MO). honduras: Dept. Atlántida, in forest foothills back to Ceiba, leg.: T. G. Yuncker et al. 8797, 04.08.1938. (MO). - Dept. Atlántida, Vegas de Juan Leandra, $10 \mathrm{~km}$ E de Ceiba, leg.: H. Martinez 189, 03.05.1980. (MO). - Distr. Atlántida, Cordillera Nombre de Díos, $5 \mathrm{~km}$ N of path on road from Las Flores to La Ceiba, leg.: S. Blackmoore \& M. Chorley 4108, 23.05.1987. (MO). - Distr. Yoro, 7 km NE of Santa Rita on road to Negrita, leg.: W. E. Harmon \& J. D. Dwyer 3910, 14.08.1970. (MO). - Distr. Yoro, alrededores de Santa Rita de Yoro, leg.: J. Mancillas \& J. Henandez R. 1088, 21.04.1971. (MO). - Ravins E of Texiquat river, 1-2 km NW of aldea La Aurora, coord.: $15^{\circ} 30^{\prime} \mathrm{N}, 87^{\circ} 26^{\prime} \mathrm{W}$, alt. 200-500 m, leg.: D. L. Hazlett, A. E. Brant \& R. Zúniga 8028, 09.04.1994. (MO). mexico: Chiapas, Mpio. Las Margaritas, low ridges at the confluence of the Rio Ixcá with the Rio Lacantum, on the Guatemalan boarder, alt. 300 m, leg.: D. E. Breedlove \& E. McClintock 34257, 14.03.1973. (MO). Chiapas, Mpio. Ocosingo, $8 \mathrm{~km}$ al S de Benemérito de la Américas, camino a Flor de Cacao, en zona de Marquéz de Comillas, alt. 150 m, leg.: E. Martinez S. 11633, 08.04.1985. (MEXU, MO). - Chiapas, $15 \mathrm{~km}$ al S de Palenque, sobre la carretera Palenque-Ocosingo, leg.: E. \& H. Cabrera 8139, 06.04.1985. (MEXU, MO). - Chiapas, Mpio. Palenque, ridge of 6-12 km S of Palenque, on the road to Ocosingo, alt. $300 \mathrm{~m}$, leg.: D. E. Breedlove 26552, 27.07.1972. (MO). - Chiapas, $3 \mathrm{~km}$ al E de la carretera Ocosingo-Palenque, camino a las cascadas de Agua Azul, leg.: E. \& H. Cabrera, \& E. Martinez 1903, 03.03.1982. (MEXU, MO). - Chiapas, Mpio. Palenque, side road to Agua Azul, $60 \mathrm{~km} \mathrm{~S}$ of Palenque, alt. 520 m, leg.: D. E. Breedlove 50844, 13.04.1981. (MO). - Chiapas, Mpio Ocosingo, Centro Archeologico Bonampak, alt. 350 m, leg.: J. Meaves, M. Soto \& R. Durán B-282, 05.02.1982. (MEXU, MO). - Chiapas, on limestone ridge of the valley to the NE of Bonampak, alt. $670 \mathrm{~m}$, leg.: W. S. Hoover 13.03.1975. (MO). - Chiapas, Mpio. Ocosingo, $8 \mathrm{~km}$ al NW de la Boca Lacantum, camino a Palenque, alt. 200 m, leg.: E. Martinez S. 11838, 09.04.1985. (MEXU, MO). - Chiapas, Mpio. Ocosingo, $15 \mathrm{~km}$ al NW de la Boca Lacantum, camino a Palenque, alt. $220 \mathrm{~m}$, leg.: E. Martinez S, 11890, 10.04.1985. (MEXU, MO). - Chiapas, Mpio. Ocosingo, 8 km NW of Bonampak, Lacanja-Cangayab at the Rio Lacanja, alt. 350 m, leg.: G. Davidse et al. 20513, 14-15.05.1982. (MO). - Chiapas, Mpio. Ocosingo, en campamento Cofolasa a $24 \mathrm{~km}$ al SE de Crucero Corozal, camino Boca Lacantum, alt. 200 m, leg.: E. Martinez S. 11201, 25.02.1985. (MEXU, MO). - Chiapas, Mpio. Ocosingo, El Crucero Corozal sobre el camino Palenque-Boca Lacantum, leg.: E. Martinez S. 11513, 05.04.1985. (MEXU, MO). - Chiapas, Mpio. Ocosingo, en el Ejido el Tumbosobre el camino Chancala-Monte Líbano, alt. 700 m, leg.: E. Martinez S. 
17552, 10.04.1986. (MEXU, MO). - Chiapas, Mpio. Ocosingo, stream near the settlement of Lacanjá, alt. 350 m, leg.: D. E. Breedlove 34534, 02.04.1973. (MO). - Tabasco, Juárez, Tenosique, leg.: E. Matuda 3392, 14-16.06.1939. (MO).

Arachnothryx standleyana (A. R. Molina) Borhidi, Acta Bot. Hung. 33: 302. (1987). Basionym: Rondeletia standleyana A. R. Molina, Ceiba 1: 262. (1951).

Arachnothryx stenostachya (Standl.) Borhidi, Acta Bot. Hung. 33: 303. (1987). Basionym: Rondeletia stenostachya Standl., Publ. Field Mus. Nat. Hist., Bot. Ser. 18: 1372. (1938). = Gonzalagunia brenesii (Standl.) Standl. et Steyerm.

Arachnothryx tacanensis (Lundell) Borhidi, Acta Bot. Hung. 33: 303. (1987). Basionym: Rondeletia tacanensis Lundell, Bull. Torr. Bot. Club 66: 603. (1939).

Specimens examined: Mexico: Chiapas, Mpio. Motozintla de Mendoza, forest near Niquivil at the junction with the small sideridge to Cerro Boquerón, alt. $2600 \mathrm{~m}$, leg.: D. E. Breedlove 42805, 16.12.1976. (MO). - Chiapas, hills E of Union Juárez, lower slopes of volcano Tacana, coord.: $15^{\circ} 06^{\prime} \mathrm{N}, 9^{\circ} 05^{\prime} \mathrm{W}$, alt. 1700-2300 m, leg.: J. S. Miller et al. 2642, 03.05.1987. (MO). - Chiapas, Mpio. Union Juárez, SE side of volcano Tacana, above Talquián, 2700 m, leg.: D. E. Breedlove 24369, 05.03.1972. (MEXU, MO). - Ibid., alt. $2200 \mathrm{~m}$, leg.: D. E. Breedlove \& F. Almeda 47724, 23.11.1980. (MO). - Chiapas, Mpio. Union Juárez, cloud forest on SW slope of volcano Tacana above Talquián, alt. $2200 \mathrm{~m}$, leg.: D. E. Breedlove 42575, 13.12.1975. (MO). - Chiapas, Mpio. Union Juárez, en el camino entre Talquian y la cima del volcán Tacana, alt. 1600-2400 m, leg.: E. Martinez S., O. Téllez \& G. Perez J. 13264, 19.06.1985. (MEXU, MO). - Ibid., alt. 1600 m, leg.: E. Martinez S. et al. 14137, 20.10.1985. (MEXU, MO). - Chiapas, Mpio. Union Juárez, Volcán Tacana, entre Chiquihuites y la cima por paso del gato, alt. $2400 \mathrm{~m}$, leg.: E. Martinez S. et al. 20507, 29.04.1987. (MEXU, MO). - Chiapas, Mpio Union Juárez, Volcán Tacana entre Talquián y Toniná, alt. 1700-2700 m, E. M. Martinez S. et al. 20763, 07.05.1987. (MEXU, MO).

Arachnothryx taylorae (Lorence) Borhidi, Acta Bot. Hung. 38: 140. 1993-94 (publ. 1995). Basionym: Rondeletia taylorae Lorence in W. C. Burger \& C. M. Taylor, Fieldiana, Bot., nov. ser., 33: 300. (1993).

Specimens examined: Costa Rica: Prov. Limón, Cantón de Limon, El Progreso, Fila Matama, Valle de la Estrella, coord.: 9 $47^{\prime} 18^{\prime \prime}$ N, 8308'45" W, alt. 1350 m, leg.: G. Herrera \& A. Chacón 2656, 19.04.1989. (MO, isotype). - Prov. Limón, Cantón de Limón, Cerro Muchilla, Fila Matama, Cordillera de Talamanca, coord.: 9 $46^{\prime} 50^{\prime \prime} \mathrm{N}, 83^{\circ} 05^{\prime} 30^{\prime \prime} \mathrm{W}$, alt. 800 m, leg.: R. Robles \& A. Chacón 2664, 05.04.1989. (MO). - Prov. Limón, Cantón de Limón, Cerro Muchilla, Fila Talamanca, coord.: 9 $47^{\prime} 50^{\prime \prime}$ N, 8305'30” W, alt. 850 m, leg.: R. Robles \& A. Chacón 2670, 06.04.1989. (MO). - Prov. Limón, Cantón de Talamanca, Braxi Alto Lari, Quivut, coord.: $9^{\circ} 23^{\prime} 50^{\prime \prime} \mathrm{N}, 8^{\circ} 05^{\prime} 20^{\prime \prime} \mathrm{W}$, alt. 1300-1500 m, leg.: R. Aguilar \& H. Schmidt 1116, 15.03.1992. (MO). 
Arachnothryx tenorioi (Lorence) Borhidi, Acta Bot. Hung. 38: 140. 1993-94 (publ. 1995).

Basionym: Rondeletia tenorioi Lorence, Novon 1: 156. (1991).

Specimens examined: Mexico: Oaxaca, Distr. Juxtlahuaca, $19 \mathrm{~km}$ al SW de Juxtlahuaca y a $5 \mathrm{~km}$ en la misma dirección de Santa María Asunción, leg.: P. Tenorio \& R. C. Torres 184, 04.04.1982. (Isotype: MO, holotype: MEXU!).

Arachnothryx thiemei (Donn.Sm.) Borhidi, Acta Bot. Hung. 28: 69. (1982). Basionym: Rondeletia thiemei Donn.Sm., Bot. Gaz. 42: 299. (1906).

Specimens examined: El Salvador: Dept. Ahuachapán, El Imposible, San Benito, lado E de Lechera, coord.: $13^{\circ} 49^{\prime}$ N, $8^{\circ} 56^{\prime}$ W, leg.: E. Sandoval 440, 15.06.1992. (MO). - Dept. Ahuachapán, El Imposible, San Francisco Menendez, sendero Pena Blanca Lindero Mejía, coord.: $13^{\circ} 49^{\prime} \mathrm{N}, 89^{\circ} 56^{\prime} \mathrm{W}$, leg.: I. Martinez s. n., 08.06.1994. (MO). - Dept. Ahuachapán, El Imposible, San Francisco Menendez, montana El Balsamar, coord.: $13^{\circ} 49^{\prime} \mathrm{N}, 89^{\circ} 56^{\prime} \mathrm{W}$, leg.: F. Lopez s. n., 08.08.1994. (MO). - Dept. Ahuachapán, Parque Nacional El Imposible, coord.: $13^{\circ} 49^{\prime} \mathrm{N}, 89^{\circ} 56^{\prime} \mathrm{W}$, leg.: A. Sermeno s. n., 16.06.1990. (MO, with fl.!). Guatemala: Dept. Santa Rosa, along hwy CA-1 14 miles W of Jutiapa, alt. 4000 ft, leg.: D. Dunn, C. Cziekanowski \& M. Pennell 23012, 26.07.1978. (MO). Honduras: Dept. Cortés, Cordillera de Merendón, 20 km al N de San Pedro Sula, orillas del Rio Zapotal, alt. 300 m, leg.: G. Padilla 25. (MO). Dept. Cortés, Fadas humedas de la montana Santa Ana, matorrales humedos del Rio Santa Ana, alt. 210 m, leg.: A. Molina R. 3608, 06.12.1950. (MO).

Arachnothryx torresii (Standl.) Borhidi, Acta Bot. Hung. 33: 303. (1987). Basionym: Rondeletia torresii Standl., J. Wash. Acad. Sci. 18: 163. (1928). = Javorkaea torresii (Standl.) Borhidi.

Arachnothryx tuxtlensis (Lorence et Cast.-Campos) Borhidi, Acta Bot. Hung. 35: 311. (1989). Basionym: Rondeletia tuxtlensis Lorence et Cast.-Campos, Biotica 13: 148. (1988).

Specimens examined: Mexico: Veracruz, Bastonal-Sierra Santa Marta road, ca $14 \mathrm{~km} \mathrm{E}$ of Lago Catemaco, alt. 700-800 m, leg.: A. Gentry \& E. Lott, 32441, 29.05.1981. (MO). Veracruz, Los Tuxtlas, Mpio. Catemaco, Bastonal, $12 \mathrm{~km}$ al NE de Catemaco, carretera Catemaco-Tebanca, alt. 950 m, leg.: S. Sinaca Colín 155, 30.07.1985. (MEXU!, MO). Veracruz, Los Tuxtlas, Mpio. Catemaco, Bastonal $8 \mathrm{~km}$ al NE de Tebanca, carretera Catemaco-Tebanca, alt. 1200 m, leg.: S. Sinaca Colín 772, 05.06.1986. (MO). - Veracruz, Mpio. Mecayapan, Volcan San Martin Pajapan al S de Ejido La Valentina, alt. 700 m, leg.: J. I. Calzada 11183, 08.05.1985. (MO, isotype). - Veracruz, Mpio. Soteapan, selva perennifolia, coord.: $18^{\circ} 21^{\prime} \mathrm{N}, 94^{\circ} 55^{\prime}$ W, alt. 1280 m, leg.: P. Acosta \& C. Gonzalez R. 1360, 20.09.1986. (MO).

Arachnothryx urophylla (Standl. et L. O. Williams) Borhidi, Acta Bot. Hung. 38: 141. 1993-94 (publ. 1995). Basionym: Rondeletia urophylla Standl. et L. O. Williams, Phytologia 26: 129. (1973). 
Arachnothryx uxpanapensis (Lorence et Cast.-Campos) Borhidi, Acta Bot. Hung. 35: 311. (1989). Basionym: Rondeletia uxpanapensis Lorence et Cast.-Campos, Biotica 13: 150. (1988). = Javorkaea uxpanapensis (Lorence et Cast.-Campos) Borhidi.

Arachnothryx villosa (Hemsl.) Borhidi, Acta Bot. Hung. 28: 69. (1982). Basionym: Rondeletia villosa Hemsl., Diag. Pl. Nov. Mexic., p. 27. (1879). Specimens examined: Mexico: Chiapas, 6 miles N of Ixtlacomitán, alt. ca $1000 \mathrm{ft}$, coord.: $17^{\circ} 20^{\prime} \mathrm{N}, 9^{\circ} 01^{\prime} \mathrm{W}$, leg.: G. L. Webster \& S. Lynch 17738, 07.08.1942. (MO). Chiapas, Mpio. Palenque, $50 \mathrm{~km}$ SW of Palenque, on road to Ocosingo, near colonia Ursulo Galvano, alt. 370 m, leg.: D. E. Breedlove 47356, 09.11.1980. (MEXU, MO). - Chiapas, Mpio. Solosuchiapa, 3-5 km above Solosuchiapa, along road to Tapilula, alt. $450 \mathrm{~m}$, leg.: D. E. Breedlove 26437, 26.07.1972. (MO). - Guatales, leg.: Liebmann 11832, 1941-43. (MO). Mpio. Acayucán, Ocotal Chico, $20 \mathrm{~km}$ al N de San Pedro Soteapán, alt. $600 \mathrm{~m}$, leg.: H. Guevara n. 3. (MO). - Oaxaca, along hwy 175, between Valle Nacional and Oaxaca, coord.: $17^{\circ} 44^{\prime}$ N, 96¹9’ W, alt. 705 m, leg.: T. B. Croat \& D. P. Hannon 65548, 21.02.1987. (MO). Oaxaca, Distr. de Choapán, La Chinantla, 5-10 km al NE de la Ermita, o sea, 15-20 km al NE de Santiago Choapán, leg.: D. H. Lorence, R. C. Torres \& A.García M. 4402, 12.11.1983. (MEXU, MO). - Oaxaca, Distr. Ixtlán, Mpio. Comaltepec, Puerto Eligio, alt. ca $700 \mathrm{~m}$, coord.: $17^{\circ} 41^{\prime}$ N, 96²17' W, leg.: E. Lopez García 23, 01.09.1987. (MEXU, MO). - Oaxaca, Distr. Tuxtepec, Rio Uloapán, a 8 km NE de San Bartolo Ayautla, leg.: R. T. Cedillo \& R. C. Torres 1901, 08.09.1981. (MEXU, MO). - Oaxaca, Mpio. Matías Romero, $3.3 \mathrm{~km}$ al E de centro de la Cuauhtemoc, sobre la terraceriade la Laguna cerca del puente Arrozal, coord.: $17^{\circ} 07^{\prime} \mathrm{N}$, 9451'45" W, alt. 90 m, leg.: T. Wendt, A. Villalobos, E. Lott \& A. Navarrete 2809, 01.10.1981. (MEXU, MO). - Oaxaca, Mpio. San Felipe Usila, Cerro Verde, camino al Vainillal de José Roldán 5 km de ENE de Usila, coord.: $17^{\circ} 56^{\prime} \mathrm{N}, 96^{\circ} 30^{\prime} \mathrm{W}$, alt. $550 \mathrm{~m}$, leg.: G. I. Manriquez et al. 3756, 01.10.1992. (MO). - Oaxaca, Mpio. Santa María Chimalapa, Arroyo Concha, ca 12 $\mathrm{km}$ al O de Santa María, por la vereda a Matías Romero, coord.: $16^{\circ} 51^{\prime} \mathrm{N}, 94^{\circ} 47^{\prime} \mathrm{W}$, alt. 300 m, leg.: H. Hernandez G. 1501, 09.09.1985. (MEXU, MO). - Oaxaca, Mpio. Santa María Chimalapa, ca $15 \mathrm{~km}$ al ESE de Santa María por la vereda a Arroyo Plata, loma al de Rio Milagro, coord.: $16^{\circ} 52^{\prime}$ N, 94³7' W, alt. 400 m, leg.: H. Hernandez G. 1440, 03.09.1985. (MEXU, MO). - Oaxaca, Santa María Chimalapa, ca $7 \mathrm{~km}$ al E de Santa María, por la vereda al Arroyo Sardina, coord.: 16 $55^{\prime}$ N, 94 ${ }^{\circ} 38^{\prime}$ W, alt. 250 m, leg.: H. Hernandez G. 302, 06.08.1984. (MEXU, MO). - Oaxaca, Santa María Chimalapa, filo del Cerro al E del Arroyo Monterico, ca $20 \mathrm{~km}$ al E de Santa María, coord.: 16 $55^{\prime}$ N, $94^{\circ} 42^{\prime}$ W, alt. 400-500 m, leg.: H. Hernandez G. \& C. Gonzalez L. 1580, 20.09.1985. (MO). - Oaxaca, Santa María Chimalapa, Rio Milagros, cerca de la embocadura del Arroyo Sangre, ca $2 \mathrm{~km}$ al SE de Santa María, coord.: $16^{\circ} 54^{\prime} \mathrm{N}, 94^{\circ} 40^{\prime} 30^{\prime \prime} \mathrm{W}$, alt. 200 m, leg.: C. Gonzalez L. 6, 31.08.1985. (MO). - Tabasco, Mogoxpa, Mpio. Teapa, 50 m, leg.: F. Ventura A. 20601, 29.11.1983. (MEXU, MO). Veracruz, Bastonal-Sierra Santa María road, ca $14 \mathrm{~km}$ al E de Lago Catemaco, alt. 700-800 m, leg.: A. Gentry \& E. Lott 32412, 29.05.1981. (MEXU, MO). - Veracruz, Mpio de San Andrés Tuxtla, poblado de Laguna Escondida, al $5 \mathrm{~km}$ de la Estación de Biología Tropical, alt. 170 m, leg.: J. Ismael Calzada 1412, 31.07.1974. (MEXU, MO). - Veracruz, Mpio. Soteapan, Ejido Santa María, camino a La Ventana, coord.: $18^{\circ} 22^{\prime}$ N, $94^{\circ} 54^{\prime}$ W, alt. 1050 m, leg.: R. \& P. Acosta et al. 1307, 19.09.1986. (MO). - Veracruz, San Andrés Tuxtla, al ENE de Tapalapán, Laguna Tisatal, 3 km al ENE de Tapalapán, leg.: J. H. Beaman 6202, 19.06.1972. (MO). 
Arachnothryx vulcanicola (Standl. et Steyerm.) Borhidi, Acta Bot. Hung. 33: 303. (1987). Basionym: Rondeletia vulcanicola Standl. et Steyerm., Publ. Field Mus. Nat. Hist., Bot. Ser. 22: 291. (1940). = Arachnothryx secundiflora (B. L. Robins.) Borhidi.

Arachnothryx wendtii (Lorence et Cast.-Campos) Borhidi, Acta Bot. Hung. 35: 311. (1989). Basionym: Rondeletia wendtii Lorence et Cast.-Campos, Biotica 13: 154. (1988).

Specimen examined: Mexico: Veracruz, Mpio. de Hidalgotitlán, zona Uxpanapa, Rio Solosuchil, a 5-6 km al ESE de Hermanos Cedillo, alt. 150 m, leg.: D. H. Lorence et al. 3971, 28.03.1982. (MO).

Arachnothryx yucatanensis (Lundell) Borhidi, Acta Bot. Hung. 35: 311. (1989). Basionym: Rondeletia yucatanensis Lundell, Wrightia 5: 329. (1976). = Arachnothryx leucophylla (Kunth) Planch.

Arachnothryx zolleriana (Standl. et Steyerm.) Borhidi, Acta Bot. Hung. 33: 303. (1987). Basionym: Rondeletia zolleriana Standl. et Steyerm., Publ. Field Mus. Nat. Hist., Bot. Ser. 22: 292. (1940). = Arachnothryx chiapensis (Brandegee) Borhidi.

Javorkaea Borhidi et J. Komlódi, Acta Bot. Hung. 29: 16. (1983). Type: Rondeletia hondurensis Donn.Sm.

Tropical trees and shrubs, twigs and leaves hirsute with strigillous hairs, stipules ending in long acuminate tip; inflorescence terminal cymose-corymbose; calyx zygomorphic 4-5(-7)-lobed, 1-2 lobes twice as broad as the others, deeply nerved. Corolla radial or slightly zygomorphic 4-5(-7)-lobed, sometimes one lobe labium-like with undulate margin, tube villous outside, pilose inside near the base; orifice open, naked, lobes hairy inside at the base and/or in the sinuses between the lobes. Ovary elliptic to oblong, annular disc entire, naked. Pollen 4-colporate, pori and colpi not at the same place, exine punctitegillate. Capsule elliptic or oblong, septicidal, laterally compressed, placenta elliptic thin, plate, with a punctiform insertion to the upper part of the septum, slightly concavious, pointed, not sulcate, seeds wingless, flattened, angulate with lateral insertion, cells with thick doubled perpendicular walls without ornamentation, horizontal ones deeply concavious, without or with obsolete ornamentation.

About 5 species in Mexico and Central America. 
Javorkaea chaconii (Lorence) Borhidi, Acta Bot. Hung. 43: 41. (2001). Basionym: Rondeletia chaconii Lorence in W. C. Burger and C. M. Taylor, Fieldiana, Bot., nov. ser., 33: 296. (1993).

Specimens examined: Costa Rica: Alajuela, Reserva Biologica Monteverde, Rio Penas Blancas, Laguna Poco Sol, coord.: $10^{\circ} 21^{\prime} \mathrm{N}, 84^{\circ} 40^{\prime} \mathrm{W}$, alt. $950 \mathrm{~m}$, leg.: E. Bello 1080, 08.08.1989. (CR, MO). - Alajuela, Reserva Biologica Monteverde, Bosque Eterno de los Ninos, Salazar, coord.: $10^{\circ} 20^{\prime}$ N, $84^{\circ} 41^{\prime}$ W, alt. 900 m, leg.: E. Bello 2082, 27.03.1990. (CR, MO). - Prov. Heredia, Braulio Carrillo cantón de Sarapiquí, Juez de Ceibo, on leach crest $250 \mathrm{~m}$ of transect trail, primary premontane wet forest, coord.: $10^{\circ} 17^{\prime} 45^{\prime \prime} \mathrm{N}, 84^{\circ} 04^{\prime} 37^{\prime \prime} \mathrm{W}$, alt. 765 m, leg.: B. Boyle \& A Espino 2953, 20.03.1994. (MO). - Prov. Heredia, pasturas bosque socolado Finca de Bernardo Gómez, Magsasay, alt. 700 m, leg.: I. A.Chacón 177, 01.01.1983. (MO, isotype). - Prov. Heredia, primary forest near Rio Peje and Rio Sardinalito, atlantic slope of Volcán Barba, coord.: $10^{\circ} 17^{\prime} 30^{\prime \prime} \mathrm{N}, 84^{\circ} 04^{\prime} 30^{\prime \prime} \mathrm{W}$, alt. 700-800 m, leg.: M. H. Grayum 6735, 03.04.1986. (CR, MO).

Javorkaea hondurensis (Donn.Sm.) Borhidi et J. Komlódi, Acta Bot. Hung. 29: 16. (1983). Basionym: Rondeletia hondurensis Donn.Sm., Bot. Gaz. 27: 335. (1899).

Javorkaea macrocalyx (Standl. et Steyerm.) Borhidi, Acta Bot. Hung. 43: 50. (2001). Basionym: Rondeletia macrocalyx Standl. et Steyerm., Publ. Field Mus. Nat. Hist., Bot. Ser. 23: 254. (1943). Synonym: Arachnothryx macrocalyx (Standl. et Steyerm.) Borhidi, Acta Bot. Hung. 33: 302. (1987).

Specimens examined: Mexico: Oaxaca, 6 miles W of Valle Nacional on highway 176 between Tuxtepec and Oaxaca, steep forested ravine, alt. $660 \mathrm{~m}$. leg.: J. Croat 39746 (MO 2601015). - Oaxaca, 5 km al NE de Puerto Eligio, alt. 900 m, leg.: Cedillo, R. T. \& Torres R. C. 2398 (MEXU, XAL).

Javorkaea megalantha (Lorence) Borhidi, Acta Bot. Hung. 40: 16. 1996-97. (publ. 1999). Basionym: Rondeletia megalantha Lorence, Novon 4: 132-134. (1994).

Javorkaea scabra (Hemsl.) Borhidi, Acta Bot. Hung. 43: 38. (2001). Basionym: Rondeletia scabra Hemsl., Diag. Pl. Nov. Mexic., p. 29. (1879). Synonyms: Sommera acuminata Oerst. ex Standl., J. Wash. Acad. Sci. 17: 340. (1927), Rondeletia acuminata (Oerst. ex Stand1.) Lorence et Cast.-Campos, Biotica 13: 148. (1988), Arachnothryx acuminata (Oerst. ex Standl.) Borhidi, Acta Bot. Hung. 35: 310. (1989), Javorkaea acuminata (Oerst. ex Standl.) Borhidi in Borhidi et al., Acta Bot. Hung. 40: 18. 1996-97 (publ. 1999).

Specimens examined: Mexico: Oaxaca, Mpio Comaltepec, Distr. Ixtlán, $5 \mathrm{~km}$ al O de La Esperanza, carr. Tuxtepec-Oaxaca, coord.: $17^{\circ} 36^{\prime}$ N, $96^{\circ} 24^{\prime} \mathrm{W}$, alt. $1760 \mathrm{~m}$, leg.: R. C. Torres \& E. Martinez 11293. (MEXU, XAL). - Oaxaca, Mpio Comaltepec, Distr. Ixtlán, 2 km 
al SO de La Esperanza, carr. Tuxtepec-Oaxaca, coord.: $17^{\circ} 37^{\prime}$ N, 96 $23^{\prime}$ W, alt. 1700 m, leg.: R. C. Torres \& L. A. Cortes 11675. (MEXU, XAL). - Oaxaca, $3.6 \mathrm{~km}$ al SO de La Esperanza, carr. Tuxtepec-Oaxaca, alt. 1700 m, leg.: R. Torres 11690, 24.02.1988. (MEXU). - Oaxaca, 13 km al SO de La Esperanza, carr. Oaxaca-Tuxtepec, leg.: R. Torres 10937, 17.10.1987. (MEXU). - Oaxaca, 13 km al N de La Esperanza, Distr. Ixtlán, alt. 1900 m, leg.: A. Garcia et al. 3045, 09.04.1987. (MEXU). - Oaxaca, $31 \mathrm{~km}$ al Sur de Valle Nacional, camino a Oaxaca, E. Martinez et al. 8797, 22.11.1984. (MEXU, 2 spec.). - Oaxaca, $5 \mathrm{~km}$ al NE de Puerto Eligio, alt. 900 m, leg.: R. Cedillo \& R. Torres 2398, 02.06.1983. (MEXU). - Oaxaca, 6.5 km al Sur de Santa Cruz Tepetotutla, alt. 2040 m, leg.: A.Rincón Gutierrez et al. 218, 22.12.1993. (MEXU) - Oaxaca, Arroyo Lumbre $3 \mathrm{~km}$ al Este de Santa Cruz Tepetotutla, alt. $780 \mathrm{~m}$, leg.: M. del Castillo et al. 1588, 05.02.1993. (MEXU). - Oaxaca, carretera Oaxaca-Tuxtepec, $111 \mathrm{~km}$, leg.: T. McDougall, 25.11.1963. (MEXU, 2 spec.). - Oaxaca, Distr. Ixtlán, Sierra de Juárez, ruta 175 Tuxtepec a Oaxaca, km 75 a La Esperanza, leg.: R. Cedillo \& D. Lorence 924. (MEXU, XAL). - Oaxaca, Mpio San Felipe Usila, $3.5 \mathrm{~km}$ al S de Santa Cruz Tepetotutla, coord.: 17³9'32" N, 96 32'46" W, alt. 2200 m, leg.: C. Gaillardo H. et al. 962. (MEXU, XAL, 2 spec.). - Oaxaca, Mpio San Felipe Usila, $5.6 \mathrm{~km}$ al S de Santa Cruz Tepetotutla, coord.: $17^{\circ} 41^{\prime} 18^{\prime \prime}$ N, $96^{\circ} 31^{\prime} 04^{\prime \prime}$ W, alt. 1970 m, leg.: C. Gaillardo H. et al. 929. (MEXU, XAL). - Oaxaca, Mpio San Felipe Usila, $6.5 \mathrm{~km}$ al S. de Santa Cruz Tepetotutla, coord.: $17^{\circ} 40^{\prime} 50^{\prime \prime} \mathrm{N}, 96^{\circ} 32^{\prime} 58^{\prime \prime} \mathrm{W}$, alt. $2040 \mathrm{~m}$, bosque mesófilo montano, leg.: C. Gaillardo H. et al. 1058, 04.04.1994. (MEXU, XAL). Oaxaca, Puerto Antonio, $5.3 \mathrm{~km}$ al N de Vista Hermosa, carr. Oaxaca-Tuxtepec, alt. $1230 \mathrm{~m}$, leg.: P. Tenorio \& R. Torres 11024, 09.03.1986. (MEXU). - Oaxaca, Puerto San Antonio, entre Metates y La Esperanza, carr. Tuxtepec-Oaxaca, alt. 1250 m, leg.: A. Garcia M. et al. 1830, 04.08.1985. (MEXU). - Oaxaca, Sierra de Juárez, Rancho La Primavera rumbo al Rio Faisán, alt. 1600 m, leg.: G. Perez Cruz C-103, 01.04.1980. (MEXU). - Oaxaca, Sierra de Juárez, ruta 175, Tuxtepec a Oaxaca, 4 km al SO de La Esperanza, alt. $1700 \mathrm{~m}$, leg.: D. Lorence \& R. Torres 4016, 14.04.1982. (MEXU). - Ibid., D. Lorence 4176, 28.05.1983. (MEXU). - Ibid., D. Lorence 4264, 04.06.1983. (MEXU). - Oaxaca, $3.1 \mathrm{~km}$ al Sur de Santa Cruz Tepetotutla, alt. $2200 \mathrm{~m}$ leg.: C. Gaillardo Hernandez et al. 847, 25.02.1994. (MEXU). - Ibid., alt. 2010 m, leg.: C. Gaillardo Hernandez et al. 1219, 31.10.1994. (MEXU). - Oaxaca, $39 \mathrm{~km}$ al S de Valle Nacional sobre la carr. Oaxaca, alt. 1750 m, leg.: Rzedowski 33835, alt. 1800 m, 27.12.1975. (MEXU). Ibid., leg.: Rzedowski 33902, 22.02.1976. (MEXU).

Javorkaea torresii (Standl.) Borhidi, comb. nova hoc loco. Basionym: Rondeletia torresii Standl., J. Wash. Acad. Sci. 18: 163. (1928). Synonym: Arachnothryx torresii (Standl.) Borhidi, Acta Bot. Hung. 33: 303. (1987).

Specimens examined: Costa Rica: Alajuela, Viento Fresco, alt. 1600-1900 m, leg.: P. C. Standley \& R. Torres 47839, 13.02.1926. (MO, isotype). - Prov. Alajuela, en fila cerca de división continetal Ventana, alt. 1520-1550 m, leg.: V. J. Dryer 1161, 26.01.1977. (MO). Prov. Alajuela, Monteverde, Cordillera de Tilarán, alt. 1520-1580 m, leg.: V. J. Dryer 256.

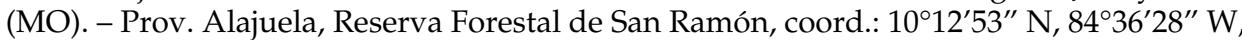
alt. 800-1000 m, leg.: G. Herrera C. \& V. Mora 185, 03.11.1986. (MO). - Guanacaste, El Dos de Tilarán, $4 \mathrm{~km} \mathrm{~N}$ Cerro La Chirripa, atlantic slope and forest, alt. $1000 \mathrm{~m}$, coord.: $10^{\circ} 25^{\prime} \mathrm{N}$, $84^{\circ} 50^{\prime}$ W, leg.: W. A. Haber, E. Bello \& L. Lierheimer 4395, 12.04.1986. (MO). - Prov. Guanacaste, Monteverde cloud forest, coord.: $10^{\circ} 48^{\prime} \mathrm{N}, 84^{\circ} 50^{\prime} \mathrm{W}$, alt. $1550 \mathrm{~m}$, leg.: A. Gentry et al. 12.07.1990. (MO). - Prov. Heredia, $9 \mathrm{~km} \mathrm{SE}$ of Virgen del Socorro, $9 \mathrm{~km}$ al E de Isla Bonita, coord.: $10^{\circ} 14^{\prime} \mathrm{N}, 84^{\circ} 05^{\prime} \mathrm{W}$, alt. $1530 \mathrm{~m}$, leg.: B. A. Loisell 221, 01.1987. (MO). - Prov. Heredia, Cantón de Barva, La Legua, Finca Montreal, between the headwaters of Rio del 


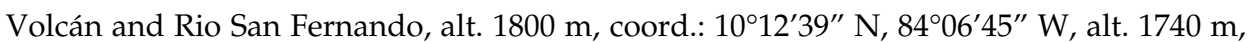
leg.: B. Boyle 1075, 09.10.1992. (MO). - Prov. Heredia, headwaters of Rio Santo Domingo, ca $3 \mathrm{~km}$ E of San Rafael de Vara Blanca, N slope of the Volcán Barva, coord.: $10^{\circ} 11^{\prime} \mathrm{N}, 8^{\circ} 04^{\prime} \mathrm{W}$, alt. 2060-2080 m, leg.: M. H. Grayum, D. Wilson \& M. Jeffery 7134, 15.04.1985. (MO). - Prov. Heredia, NW slope of the Volcán Barva, ca $2 \mathrm{~km} \mathrm{NE}$ of Los Cartagos, coord.: $10^{\circ} 09^{\prime} \mathrm{N}, 84^{\circ} 09^{\prime}$ W, alt. 2075 m, leg.: M. H. Grayum \& G. Yatskievich 6616, 16.03.1986. (MO). - Prov. Heredia, Parque Nacional Braulio Carrillo, sendero del transecto, Cerro Las Marías to Refugio 2070 m, coord.: 10¹0'07" N, 8406'48" W, alt. 2210-2240 m, leg.: B. Boyle 836, 24.05.1992. (MO). Ibid., leg.: B. Boyle 855, 24.05.1992. (MO). - Ibid., B. Boyle 865. (MO). - Ibid., B. Boyle 927,

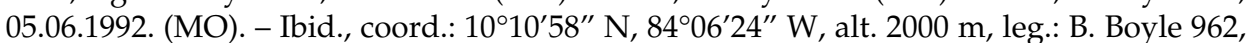
29.07.1992. (MO). - Prov. Limón, Reserva Jitoy-Cerere, bosque por el Rio Jitoy y hasta la

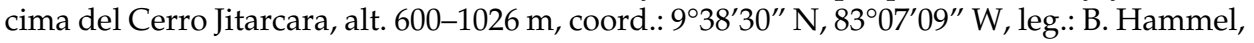
A. Chacón \& G. Herrera 17684, 03.09.1984. (MO). - Prov. Puntarenas, Cordillera de Tilarán, Monteverde, Reserve above Santa Elena, alt. 1500 m, coord.: $10^{\circ} 17^{\prime} \mathrm{N}, 84^{\circ} 47^{\prime} \mathrm{W}$, leg.: K. Barringer, E. Christenson \& B. Perez 4195, 30.07.1983. (MO). - Prov. Puntarenas, Monteverde, Reserva Biológica, leg.: L. J. Poveda 1125. (MO). - Prov. Puntarenas, Monteverde, Reserva Forestal, alt. 1550 m, coord.: $10^{\circ} 48^{\prime}$ N, 845’ W, leg.: A. Gentry 48806. (MO). - Prov. Puntarenas, Monteverde, sendero Chomogo, alt. 1500-1600 m, leg.: W. A. Haber \& E. Bello C. 2621, 07.09.1985. (MO). - Prov. Puntarenas, Rio Rits, alt. 1600 m, leg.: S. Koptur SK-88, 15.01.1949. (MO). - Puntarenas, Cantón de Puntarenas, Cordillera de Tilarán, San Luis, Cerro Banquete, camino a Surtubal, Monteverde, coord.: $10^{\circ} 15^{\prime} 25^{\prime \prime} \mathrm{N}$, 84²7'20" W, alt. 1100-1200 m, leg.: Z. Fuentes 698, 22.03.1994. (MO). - Puntarenas, margen de la Reserva Monteverde, Cerro Ojo de Agua, Finca Nemo Moya, división continental, coord.: $10^{\circ} 17^{\prime} \mathrm{N}, 84^{\circ} 46^{\prime} \mathrm{W}$, alt. 1700 m, leg.: W. Haber \& E. Bello 7030, 22.04.1987. (MO). Puntarenas, Monteverde, Cordillera de Tilarán, leg.: V. C. Ponds 264, 01.06.1984. (MO). Puntarenas, Monteverde, pacific slope, alt. $1700 \mathrm{~m}$, coord.: $10^{\circ} 18^{\prime} \mathrm{N}, 84^{\circ} 48^{\prime} \mathrm{W}$, leg.: W. A. Haber \& E. Bello 6253, 15.11.1986. (MO). - Prov. San José, Monteverde, Reserva Biológica, alt. 1500 m, leg.: W. A. Haber 521, 06.05.1981. (MO). - Dept. San José, cerro entre Cerro Chimú y Cerro Matama, alt. 1200 m, leg.: L. D. Gómez et al. 23579, 30.04.1985. (MO). - Vara Blanca de Sarapiqui, N slope of the Central Cordillera, between Poás and Barva volcanoes, alt. 1680 m, leg.: A. F. Skutch 3390, 01.1935. (MO).

Javorkaea uxpanapensis (Lorence et Cast.-Campos) Borhidi in Borhidi et al., Acta Bot. Hung. 40: 19. 1996-97 (publ. 1999). Basionym: Rondeletia uxpanapensis Lorence et Cast.-Campos, Biotica 13: 150. (1988). Synonym: Arachnothryx uxpanapensis (Lorence et Cast.-Campos) Borhidi, Acta Bot. Hung. 35: 311. (1989).

Specimens examined: Mexico: Oaxaca, Mpio. Matías Romero, Lomas al N del Rio Hamacá, pocos $\mathrm{km}$ al E del entronque con el Rio Verde, $10 \mathrm{~km}$ por camino al ESE del Aserradero La Floresta, coord.: $12^{\circ} 02^{\prime}$ N, $94^{\circ} 43^{\prime}$ W, alt. $200 \mathrm{~m}$, leg.: T. Wendt et al. 3713, 16.03.1982. (MO). - Oaxaca, Mpio. Santa María Chimalapa, Lomas al Sur del Rio Verde, ca 9 $\mathrm{km}$ al N de Santa María, por la vereda a la cabezera del Rio Verde, coord.: $16^{\circ} 58^{\prime} \mathrm{N}, 94^{\circ} 40^{\prime}$ W, alt. 400 m, leg.: H. Hernandez G. 1143, 23.04.1985. (MO). - Veracruz, Rio Marquez, a 25 km al SE, Hidalgotitlán, alt. 150 m, leg.: M. Avendado \& A. Juan 115. (XAL 21623, 21624). Veracruz, por el Rio Marquez, $25 \mathrm{~km}$ por SE del campamento Hnos. Cedillo, Hidalgotitlán, alt. $150 \mathrm{~m}$, leg.: A. Ortiz \& Martiniano 144. 0.05.1975. (XAL 21629). 
Rogiera Planch., Fl. Serres Jard. 5: tab. 442. (1849). Lectotype: Rogiera amoena Planch. designated by Borhidi, Acta Bot. Hung. 28: 67. (1982).

Shrubs or small trees with usually large, foliaceous and reflexed stipules, leaves opposite or 3-verticillate; inflorescence broad terminal, cymose-paniculate or cymose-corymbose, many-flowered; flowers 5-parted, calyx lobes small or large and foliaceous, corolla glabrous or antrorsepilose outside, densely yellow-bearded in the orifice, callosity or denticles absent; corolla tube puberulent or villous within, stamens included beneath the throat, filaments, short, puberulent, anthers elliptic, included in the bearded throat; style deeply bilobate, naked. Ovary globose, disc annular naked. Placenta sphaeroidal, little, soft with a punctiform central insertion to the septum. Ovules numerous, perpendicularly disposed to the placenta. Pollen grains sphaeroidal or subsphaeroidal, 3-colporate, exine thick, densely foveolate with tight irregular foveoles and exserted ora. Capsule globose, loculicidally dehiscent, 2-valvate. Seeds angulate or rounded not winged, with central or subcentral insertion, cells oblong, narrow with thin perpendicular walls densely ornamented by globose thickenings, horizontal walls shallowly concavious with wall-like thickenings forming areoles ornamented by globose thickenings. Basic chromosome number $\mathrm{x}=10$.

About $12-15$ species in SW United States, Mexico and Central America to Panama.

Rogiera amoena Planch., Fl. Serres Jard. 5: tab. 442. Figs 1, 2. (1849). Synonym: Rondeletia amoena (Planch.) Hemsl., Diag. Pl. Nov. Mexic., p. 26. (1879).

Specimens examined: Guatemala: Dpto Alta Verapaz, Coban. 4300 pié, leg.: H. von Türckheim (Exs. Pl. Guat. Donnel Smith 1372) (P). Mexico: Chiapas, Mpio Hiustán, 17 miles from Hwy 190, on road to Ocosingo, alt. 2080 m, leg.: P. A. Fryxell \& E. J. Lott 3294 (XAL). Chiapas, Mpio Jaltenango, Reserva del Triunfo, camino para la Finca Prusia, alt. $1350 \mathrm{~m}$. leg.: J. I. Calzada et al. 9137. (XAL 6590). - Chiapas, Mpio La Trinitaria, Lagunas de Montebello, $3 \mathrm{~km}$ desviación carr. Montebello hacia Bonanpak, coord.: $16^{\circ} 04^{\prime} \mathrm{N}, 91^{\circ} 20^{\prime} \mathrm{W}$, alt. 1530 m, leg.: J. G. Garcia F. et al. 288. (XAL 2027). - Chiapas, Mpio Pueblo Nuevo Solistahuacan, Reserva Ecologica Yerbabuena, $3 \mathrm{~km}$ del pueblo rumbo a Pichucalco, alt. 2200 m, leg.: M. B. Chazaro et al. 5340. (GUAD, XAL). - Chiapas, Mpio Tenajapa, Loc. Shishintomil, leg.: A. Shilom Ton 4749. (MEXU, XAL). - Chiapas, Mpio Tenejapa, Paredones del Cerro Cruz Ch'en, alt. 1900 m, leg.: A. Shilom Ton 4800. (MEXU, XAL). Chiapas, Mpio Tenejapa, Rio Yash, Zanal, alt. 1500 m, leg.: A. Shilom Ton 5314. (MEXU, XAL). - Guerrero, $20.1 \mathrm{~km}$ al E de Petlaltina y $68.1 \mathrm{~km}$ al E de Chilapa, camino a Tlapa Gro., alt. 170 m, leg.: R. C. Torres et al. 1932. (MEXU, XAL). - Jalisco, Mpio Cuautitlán, poblado del aserradero de Manatlán, carr. para Puerta Pesada. Sierra Manantlán, alt. 1520 m, leg.: J. I. Calzada \& G. H. Nieves 9548. (XAL 2319). - Jalisco, Mpio Cuautitlán, Res. de Biosfera 
Rancho La Joyas, Sierra de Manantlán, alt. 2100 m, leg.: M. V. \& L. V. de Puga 13075. (XAL). - Jalisco, Top of Sierra Manantlán along trail to Las Joyas $5 \mathrm{~km}$ of La Cumbre, $5 \mathrm{~km}$ SSW to WSW of Rincón de Manantlán, 16-18 km SSW of El Chante, coord.: 19³3'30"' N, 104¹4' W, alt. 2100 m, leg.: H. H. Iltis et al. 1239. (XAL, 2 spec.). - Oaxaca, Distr. Tehuantepec, Mpio. Cueva de Humboldt, $8.3 \mathrm{~km}$ al N. de la Cueva, leg.: R. Torres 9124. (MEXU, XAL). - Oaxaca, Highway 190, S of San Cristobal de las Casas and Ocosingo, 25.5 miles NE of junction with Hwy 190, alt. 1720 m, leg.: T. B. Croat 46474. (MO, XAL). nicaragua: Dept. Jinotega, Macizos de Penas Blancas, SW of Finca Manuel Estrada (El Cielo), S of Rio Gusaneras, alt. $13^{\circ} 15^{\prime} \mathrm{N}$, 8542' W, alt. 1200-1300 m, leg.: Stevens \& Krukoff 115567. (MO, XAL). - in monte Pantasma, leg.: Oersted 11678. (Mus. Bot. Haun.) (US 1315195).

Rogiera aprica (Lundell) Borhidi, Acta Bot. Hung. 33: 301. (1987). Basionym: Rondeletia aprica Lundell, Contr. Univ. Mich. Herb. 7: 54. (1942). = Rogiera cordata (Benth.) Planch.

Rogiera backhousii (Hook. f.) Borhidi, Acta Bot. Hung. 33: 301. (1987). Basionym: Rondeletia backhousii Hook. f., Bot. Mag. 103: tab. 6290. (1877).

Rogiera brachistantha (Standl. et Steyerm.) Borhidi, Acta Bot. Hung. 33: 301. (1987). Basionym: Rondeletia brachistantha Standl. et Steyerm., Publ. Field Mus. Nat. Hist., Bot. Ser. 23: 25. (1943). = Rogiera cordata (Benth.) Planch.

Rogiera breedlovei (Lorence) Borhidi, Acta Bot. Hung. 40: 16. 1996-97 (publ. 1999). Basionym: Rondeletia breedlovei Lorence, Novon 4: 129. (1994).

Specimen examined: Mexico: Chiapas, Mpio. Tenejapa, Rio Cruz Pilal, alt. 800 m, leg.: A. Shilom Ton 5295. (MEXU, XAL).

Rogiera chiapasensis (Standl.) Borhidi, Acta Bot. Hung. 33: 301. (1987). Basionym: Rondeletia chiapasensis Standl., Publ. Field Mus. Nat. Hist., Bot. Ser. 22: 54. (1940). = Gonzalagunia chiapasensis (Standl.) Standl. et Steyerm.

Rogiera cordata (Benth.) Planch., Fl. Serres Jard. 5: tab. 442b. (1849). Basionym: Rondeletia cordata Benth., Pl. Hartweg., p. 85. (1841).

Specimens examined: El Salvador: Dept. Ajuachapán, transecto 1, San Benito, El Imposible, coord.: $13^{\circ} 49^{\prime} \mathrm{N}, 8^{\circ} 56^{\prime} \mathrm{W}$, leg.: Cermeno H. S. 0016 J. P. L. 928, 12.07.1989. (MO, as R. seleriana). - Ajuachapán, El Imposible Reserve, Campana, coord.: $13^{\circ} 51^{\prime}$ N, $89^{\circ} 54^{\prime} \mathrm{W}$, alt. 1400 m, leg.: A. Mondró, G. Davidse, K. Sidwell, L. E. Escobar 2722, 24.01.1998. (B, BM, ITC, LAGU, MO). Guatemala: 5 km SE of Guatemala City, leg.: W. E. Harmon 1995, 26.02.1970. (MO). -7 km E of City Guatemala, leg.: D. E. Harmon 2253, 26.04.1973. (MO). - 
Dept. Santa Rosa, en el volcán Yumaitepec alt. 6000 ft, leg.: Heide \& Lux March 12.1892. (MO). - Prov. F. Morazán, Parque Nacional La Tigra (El Rosario), alt. 1700 m, leg.: S. Lagos-Witte, R. C. Torres \& P. R. House 15, 16.08.1987. (MO). - Quetzatenango along hwy cito, $\mathrm{N}$ of junction ca $2 \mathrm{~km}$ toward Quezaltenango, in watershed reserve INDE Santa María, km 199, coord.: $14^{\circ} 45^{\prime}$ N, $9^{\circ} 32^{\prime}$ W, leg.: T. B. Croat \& P. T. Hammen 63420, 22.01.1987. (MO). Mexico: Chiapas, Chaonil, $1 \mathrm{~km}$ al $\mathrm{N}$ en la carretera internacional, leg.: F. Gómez Santis 394, 25.09.1988. (MO). - Chiapas, Mpio Angel Albino Corso, at the base of Cerro Tres Picos, near Cerro Bola, along a logging road SW of colonia de Agrónomos Mexicanos, alt. 1500 m, leg.: D. E. Breedlove \& R. F. Thorne 30046, 10.12.1972. (MO, with fl.). - Chiapas, Mpio. Angel Albino Corso, above Finca Cuxtepec, alt. 1380 m, leg.: D. E. Breedlove 56915, 10.01.1982. (MO). - Ibid., alt. 1970 m, leg.: D. E. Breedlove 57022, 11.01.1982. (MO). Chiapas, Mpio Mapastepec, SE de la Reserva del Triunfo, tramo cupresal al Rio Sisilar, alt. 1700 m, leg.: J. I. Calzada et al. 8888. (XAL 6588). - Chiapas, Mpio Ocosingo, en Cerro Tres Picos, por la colonia Tres Picos, alt. 2200 m, leg.: E. Martinez S. \& A. Garcia 17509, 05.04.1986. (MEXU, MO: as R. brachystantha, XAL). - Chiapas, Mpio Pueblo Nuevo, $2 \mathrm{~km}$ al NW de la ciudad, leg.: E. Martinez et al. 3242. (MEXU, XAL). - Chiapas, Mpio Union Juárez, Ejido Talquián, alt. 1360 m, leg.: J. I. Calzada et al. 3702. (XAL 2005). - Chiapas, Mpio. Motozintla de Mendoza, Cerro Mozotál, $11 \mathrm{~km} \mathrm{NW}$ of the junction of the road to Motozintla, along the road to El Porvenir and Siltepec, alt. $2100 \mathrm{~m}$, leg.: D. E. Breedlove 25791, 27.06.1972. (MO, as Rogiera seleriana mihi). - Ibid., D. E. Breedlove 41694, 21.00.1976. (MO). - Chiapas, Mpio. Motozintla, road from El Rorario to Cerro Boquerón, $2 \mathrm{~km} \mathrm{~S}$ of Ojo de Agua, coord.: 1517' N, 92 $21^{\prime}$ W, alt. 1750 m, leg.: P. J. Stafford, E. J. Hampshire \& A. Reyes García 325, 08.02.1990. (MO). - Chiapas, Cerro del Boquerón, solala, leg.: C. A. Purpus, 08.1913. (MO). - Chiapas, along road to Motozintla just above Unión Juárez, lower slopes of volcán Tacaná, leg.: J. S. Miller, J. K. Myers \& E. Martinez 2710, 05.05.1987. (MO). Volcán Tacaná, cerca de Chiquihuites, alt. 1920 m, leg.: R. Hernandez M. 505, 10.02.1969. (MO). - Chiapas, Mpio. Pueblo Nuevo, 2 km NW de Pueblo Nuevo, alt. 1700 m, leg.: E. Martinez S. \& O. Téllez 3242, 11.02.1983. (MO, with fr.). - Chiapas, Mpio. San Andrés Larrainzal, summit of Uchilton, SE of Bochil, alt. 2700 m, leg.: D. E. Breedlove 26790, 03.08.1972. (MO). Chiapas, Mpio. San Andrés Larrainzal, cloud forest near summit of Chuchiltol, NE of Bochil, alt. 2700 m, leg.: D. E. Breedlove 29228, 17.10.1972. (MO). - Chiapas, San Cristóbal, along the road between Hwy $190 \mathrm{~S}$ of San Cristóbal de las Casas and Ocosingo, 25 miles E of junction with Hwy 190, alt. 1720 m, leg.: T. B. Croat 46474, 26.01.1979. (MO, 2 spec.). Chiapas, Saxchanal, Sierra Madre, leg.: E. Matuda 4314, 1-5.07.1941. (MO, US, isotype of Rondeletia aprica Lundell). - Chiapas, Mpio. Sintalapa, $10 \mathrm{~km} \mathrm{~N} \mathrm{of} \mathrm{Sintalapa,} \mathrm{coord.:} 16^{\circ} 40^{\prime}$ N, 9390’ W, alt. 1150 m, leg.: P. J. Stafford, E. J. Hampshire \& A. Reyes García 100, 110, 30.01.1990. (MO, 2 spec.). - Chiapas, Siltepec, leg.: E. Matuda S-224. (MO). - Oaxaca, San Miguel Chimalapa, $3 \mathrm{~km}$ al $\mathrm{N}$ de la colonia Rodulfo Figueroa, por el camino a Diaz Ordaz en la canada ca $1 \mathrm{~km}$ al O del camino ca $23 \mathrm{~km}$ en linea recta al N de San Pedro Tapanatepec, leg.: T. Wendt et al. 4705, 23.12.1984. (MO).

Rogiera edwardsii (Standl.) Borhidi, Acta Bot. Hung. 43: 43. (2001). Basionym: Rondeletia edwardsii Standl., Trop. Woods 37: 31. (1934). Synonym: Arachnothryx edwardsii (Standl.) Borhidi, Acta Bot. Hung. 33: 301. (1987).

Specimens examined: Honduras: Santa Cruz de Yojoa, Cortés, alt. 2000 ft, leg.: G. B. Edwards P-13A, 26.11.1933. (MO). Mexico: Chiapas, 3 km al N de Ocozocoautla, al orilla del 
camino a Mal Paso, sitio: Ocozocoautla de Espinoza, alt. 900 m, leg.: D. E. Breedlove \& L. E. Dressler 29699, 19.11.1972. (MO). - Oaxaca, Distr. Tehuantepec, al NO de Chibiza, hacía la carretera La Chiguira, alt. 1170 m, leg.: R. C. Torres \& C. Martinez 5719, 14.08.1984. (MO).

Rogiera ehrenbergii (K. Schum. ex Standl.) Borhidi, Acta Bot. Hung. 28: 67. (1982). Basionym: Rondeletia ehrenbergii K. Schum. ex Standl., N. Amer. Fl. 32: 50. (1918). = Rogiera gratissima Planch. et Linden.

Rogiera elegans Planch., Fl. Serres Jard. 5: tab. 442. (1849). = Rogiera amoena Planch.

Rogiera gratissima Planch. et Linden, Fl. Serres Jard. 15: 133. (1864). Synonym: Rondeletia gratissima (Planch. et Linden) Hemsl., Diag. Pl. Nov. Mexic., p. 25. (1879).

Specimens examined: Mexico: Chiapas, a $3 \mathrm{~km}$ al E de Tziscao, Parque Nacional de Montebello, leg.: E. Cabrera et al. 2961, 23.06.1982. (MEXU, MO). - Chiapas, a $10 \mathrm{~km}$ al E de Tziscao, sobre el acamino a Santa Elena, leg.: E. \& H. Cabrera 6099, 05.12.1983. (MEXU, MO). - Chiapas, Mpio. La Trinitaria, E of Laguna Tziscao, Monte Bello National Park. alt. 1300 m, leg.: D. E. Breedlove 35091, 13.05.1973. (MO). - Chiapas, Mpio. La Trinitaria, Pine-oak-Liquidambar forest along the Comitán River at its sunidero, Lagos de Montebello, $42 \mathrm{~km}$ NE of La Trinitaria, alt. $1300 \mathrm{~m}$, leg.: D. E. Breedlove \& R. F. Thorne 21201, 13.10.1971. (MO). - Chiapas, Mpio. La Trinitaria, Pinus-Quercus-Liquidambar forest at Lago Tziscao, Lagos de Montebello National Park, alt. 1370 m, leg.: D. E. Breedlove 58390, 09.02.1982. (MO).

Rogiera intermedia (Hemsl.) Borhidi, Acta Bot. Hung. 28: 67. (1982). Basionym: Rondeletia intermedia Hemsl., Diag. Pl. Nov. Mexic., p. 26. (1879).

Specimens examined: Mexico: Chiapas, Mpio Oxchuc, orilla de la comunidad de Oxchuc, alt. $2000 \mathrm{~m}$, coord.: 16² $47^{\prime} 31^{\prime \prime} \mathrm{N}, 9^{\circ} 20^{\prime} 42^{\prime \prime} \mathrm{W}$, leg.: A. Chamé \& A. Luna 244. (MEXU, XAL).

Rogiera langlassei (Standl.) Borhidi, Acta Bot. Hung. 28: 67. (1982). Basionym: Rondeletia langlassei Standl., N.Amer.Fl. 32: 53. 1918. = Rogiera amoena Planch.

Rogiera latifolia (Oerst.) Decne., Rev. Hortic., ser. 4, 2: 121. (1853). Basionym: Rondeletia latifolia Oerst., Vidensk. Meddel. Dansk Naturhist. Foren., Kjöbenhavn, 1852: 43. (1853). = Rogiera amoena Planch. 
Rogiera ligustroides (Hemsl.) Borhidi, Acta Bot. Hung. 28: 67. (1982). Basionym: Rondeletia ligustroides Hemsl., Diag. Pl. Nov. Mexic., p. 26. (1879).

Specimens examined: Mexico: Chiapas, Mpio La Trinitaria, Parque Nac. Montebello, arco donde se hunde el Rio, Lago de Colores, alt. 1900 m. leg.: M. B. Chazaro et al. 5541. (GUAD, XAL). - Oaxaca, Distr. Tehuantepec, Mpio Cueva de Humboldt. Cerro Picacho 8.3 $\mathrm{km}$ al $\mathrm{N}$ de la Cueva, leg.: R. C. Torres et al. 9136. (MEXU, XAL). - Veracruz, camino de herradura Aguacate (Ver.) Patlanalán, Puebla, Mpio Quimixtlán, Puebla, alt. 1800 m, leg.: M. B. Chazaro \& J. Marquez 2436. (XAL 6557). - Veracruz, carretera Orizaba a Zongolica, ca $5 \mathrm{~km}$ al Sur de San Andrés Tejapán hacía Tequila, bosque ripario de Platanus y Liquidambar, alt. 1600 m, leg.: D. Lorence \& T. P. Ramamoorthy 3895, 11.03.1982. (MEXU, MO, XAL). Veracruz, San Andrés, carretera Orizaba-Zongolica, coord.: $18^{\circ} 47^{\prime} \mathrm{N}, 97^{\circ} 03^{\prime} \mathrm{W}$, alt. $1270 \mathrm{~m}$, leg.: M. R. Rosas 667. (F, XAL 39250). - Veracruz, El Naranjito, carretera entre Orizaba y Zongolica, alt. $1600 \mathrm{~m}$, coord.: $26^{\circ} 05^{\prime} \mathrm{N}, 86^{\circ} 00^{\prime} \mathrm{W}$, leg.: M. Rosas R. 1484, 12.12.1968. (MO). Veracruz, carretera Orizaba-Tequila, $5 \mathrm{~km}$ al S de San Andrés Tejapán, leg.: T. P. Ramamoorthy 4297, 31.07.1983. (MO). - Veracruz, Cerro al O de Fortín, Torre de Microhondas, coord.: $28^{\circ} 05^{\prime} \mathrm{N}, 87^{\circ} 00^{\prime} \mathrm{W}$, alt. $800 \mathrm{~m}$, leg.: E. Lott 1207, 21.12.1970. (MO). Veracruz, Cerro de Huacapán, 2 km al N de Abzacán, leg.: M. Rosas R. 350, 07.05.1967. (MO). - Veracruz, Moyac, leg.: E. Matuda 1521, 14.05.1937. (MO). - Veracruz, Mpio Coscomatepec, Cerro La Magdalena $3 \mathrm{~km}$ al S de Tozondo, coord.: 19 $06^{\prime} 00^{\prime \prime} \mathrm{N}, 97^{\circ} 04^{\prime} 00^{\prime \prime}$ W, alt. 1700 m, leg.: J. L. Martinez \& A. Hernandez 1296. (XAL 114391). - Veracruz, Mpio Huatusco, $4 \mathrm{~km}$ antes de Elotepec, al $\mathrm{N}$ de Huatusco, coord.: $19^{\circ} 12^{\prime} \mathrm{N}, 97^{\circ} 02^{\prime} \mathrm{W}$, alt. $1650 \mathrm{~m}$, leg.: Avendado S. R. et al. 0380. (F, XAL 39147). - Veracruz, Mpio Huatusco, Camino a Tepampa, a $4 \mathrm{~km}$ de la carr. Huatusco-Coscomatepec, coord.: $19^{\circ} 10^{\prime} \mathrm{N}, 97^{\circ} 00^{\prime} \mathrm{W}$, alt. 1580 $\mathrm{m}$, bosque caducifolio, leg.: Avendado, S. R. \& J. I. Calzada 0401. (F, XAL 39148). - Veracruz, Mpio Huiloapan, Cerro de San Cristobal, coord.: $18^{\circ} 48^{\prime}$ N, $95^{\circ} 05^{\prime}$ W, alt. $1300 \mathrm{~m}$, leg.: J. I. Calzada 8579. (XAL 67968). - Veracruz, Mpio Ixhuacan de los Reyes, $8 \mathrm{~km}$ al SW of center of Toledo, $1 \mathrm{~km} \mathrm{~N}$ of turnoff to Cosautlán, coord.: 19²0'30" N, 97 $01^{\prime}$ W, leg.: M. Nee 22508. (XAL). - Veracruz, Mpio Ixhuacan de los Reyes, al Sur del poblado de Barranca Grande rio arriba, leg.: G. Castillo-Camp. et al. 5541. (MEXU, XAL). - Veracruz, Mpio Magdalena, alt. 1490 m, árbol de 7 m, leg.: V. Vazquez T. 0348. (F, XAL 39012). - Veracruz, Mpio. San Juan, Coscomatepec, El Durazno, en ladera del cerro, alt. 1600 m, leg.: F. A. Ventura 3151, 23.02.1971. (MO, XAL). - Veracruz, Mpio. de Orizaba, Cerro San Cristóbal al SE de Orizaba, leg.: D. H. Lorence 4834, 25.08.1985. (MO). - Veracruz, Sierra San Cristóbal, between ciudad Mendoza and Orizaba, alt. 1260-1400 m, leg.: T. B. Croat 39496, 27.06.1977. (MO). Veracruz, Mpio. Texhuacán, $5 \mathrm{~km}$ of SSW of Zongolica, along gravel road to Texhuacán, coord.: $18^{\circ} 39^{\prime} \mathrm{N}, 97^{\circ} 00^{\prime} \mathrm{W}$, alt. $1350 \mathrm{~m}$, leg.: M. Nee \& K. Taylor 29465, 08.02.1984. (MO). Veracruz, Poblado La Paz, carr. Coatepec-Huatusco, leg.: J. I. Calzada 2001. (XAL 38950). Veracruz, Santa Ana, leg.: A. C. Purpus 1252, 05.1905. (MO).

Rogiera macdougallii (Lorence) Borhidi, Acta Bot. Hung. 38: 140. 1993-94 (publ. 1995). Basionym: Rondeletia macdougallii Lorence, Novon 1: 144. (1991).

Specimen examined: Mexico: Oaxaca, Cerro Azul (top) N of Niltepec, alt. $7000 \mathrm{ft}$, Col.: T. MacDougall s. n., 07.03.1956. (MEXU 253131, holotype). 
Rogiera menechma Planch., Fl. Serres Jard. 5: tab. 442. (1849). Type not designated.

Rogiera nicaraguensis (Oerst.) Borhidi, Acta Bot. Hung. 43: 51. (2001). Basionym: Rondeletia nicaraguensis Oerst., Vidensk. Meddel. Dansk Naturhist. Foren., Kjöbenhavn, 1852: 43. (1853). Type: Nicaragua, Matagalpa, Segovia, in monte Pantasmo, leg.: Oersted 11825, 01.1848. (C).

Specimens examined: Honduras: Choluteca, Dept. San Marcos de Colón, El Aguacate, road to Las Delicias, ca 3 miles S of San Francisco, coord.: $13^{\circ} 21^{\prime} 26^{\prime \prime} \mathrm{N}, 86^{\circ} 54^{\prime} 22^{\prime \prime} \mathrm{W}$, alt. 1125 m, leg.: G. Davidse, M. Sousa \& J. L. Linares 35053, 17.06.1994. (MO). - Dept. Francisco Morazán: Mpio. Tegucigalpa, $2.5 \mathrm{~km}$ SE of Tegucigalpa, near El Tablón, along the water pipe. Coord.: $14^{\circ} 01^{\prime} \mathrm{N}, 87^{\circ} 09^{\prime}$ W, alt. $1020 \mathrm{~m}$, leg.: P. J. M. \& H. Maas, J. L. Linares 8468, 30.10.1996. (MO, U, with fl. \& fr.). - Dept. Francisco Morazán, alrededores de la colonia Soto, Tegucigalpa, a $900 \mathrm{~m}$ de altura, savana, leg.: N. Castro B. 82, 20.08.1978. (MO). nicaragua: Dept. Estelí, $1 \mathrm{~km}$ al $\mathrm{N}$ de San José de la Laguna, coord.: $12^{\circ} 58^{\prime} \mathrm{N}, 86^{\circ} 20^{\prime} \mathrm{W}$, alt. ca 1300 m, leg.: P. P. Moreno 16747, 24.06.1982. (MO). - Dept. Estelí, Cerro La Savana en Santa Cruz Buenavista, coord.: $12^{\circ} 58^{\prime} \mathrm{N}, 86^{\circ} 18^{\prime} \mathrm{W}$, alt. $1000 \mathrm{~m}$, leg.: P. P. Moreno 21602, 29.06.1983. (MO). - Dept. Estelí, La Laguna, a $9 \mathrm{~km}$ de la carretera panamericana, coord.: $12^{\circ} 57^{\prime} \mathrm{N}$, $86^{\circ} 20^{\prime}$ W, alt. 1280-1300 m, leg.: P. P. Moreno 21610, 29.06.1983. (MO). - Dept. Estelí, La Gavilana, coord.: $13^{\circ} 03^{\prime} \mathrm{N}, 86^{\circ} 19^{\prime}$ W, alt. $940-1000 \mathrm{~m}$, leg.: P. P. Moreno 21879, 02.08.1983. (MO). - Dept. Estelí, Quetzalcayán, al E del Cerro Tisey, coord.: $12^{\circ} 59^{\prime} \mathrm{N}, 86^{\circ} 24^{\prime} \mathrm{W}$, alt. 900-1100 m, leg.: P. P. Moreno 17802, 15.10.1982. (MO). - Dept. Managua, camino de Puertas Viejas, coord.: $12^{\circ} 35-37^{\prime} \mathrm{N}, 86^{\circ} 01-03^{\prime} \mathrm{W}$, alt. $400-500 \mathrm{~m}$, leg.: M. Araquistain 3604, 08.07.1983. (MO). - Dept. Matagalpa, Rincón del Diablo, camino a Puertas Viejas, coord.: $12^{\circ} 33^{\prime} \mathrm{N}, 85^{\circ} 53^{\prime}$ W, alt. 800 m, leg.: M. Araquistain 3697, 29.09.1983. (MO). - Dept. Matagalpa, Cerro El Nancital, al SW de Santa Rosa del Penón, carretera a Santa Bárbara, coord.: $12^{\circ} 46^{\prime} \mathrm{N}, 8^{\circ} 21^{\prime} \mathrm{W}$, alt. 300-400 m, leg.: P. P. Moreno 21574, 22.06.1983. (MO).

Rogiera pittieri (K. Schum. et K. Krause) Borhidi, Acta Bot. Hung. 28: 67. (1982). Basionym: Rondeletia pittieri K. Schum. et K. Krause in K. Schum., Bot. Jahrb. 40: 316. (1908). = Rogiera amoena Planch.

Specimen examined: Costa Rica: Leg.: H. Pittier \& T. Durand 3243. (P).

Rogiera roezlii Planch., Fl. Serres Jard. 5: tab. 442. (1849). = Rogiera amoena Planch.

Rogiera seleriana (Loes.) Borhidi, Acta Bot. Hung. 35: 311. (1989). Basionym: Rondeletia seleriana Loes., Verh. Bot. Verein. Brandenb. 65: 105. (1913). = Rogiera gratissima Planch. et Linden.

Rogiera stenosiphon (Hemsl.) Borhidi, Acta Bot. Hung. 28: 67. (1982). Basionym: Rondeletia stenosiphon Hemsl., Diag. Pl. Nov. Mexic., p. 26. (1879). 
Specimens examined: Mexico: Chiapas, $17 \mathrm{~km}$ al S de la desviación a las cascadas de Agua Azul, por la carr. Ocosingo-Palenque. Selva alta. Leg.: E. \& H. de Cabrera 8137. (MEXU, XAL). - Chiapas, Mpio Chilan, carr. de Palenque a Ocosingo 73 km, leg.: A. Shilom Ton 5530. (MEXU, XAL). - Chiapas, Mpio La Trinitaria, 36-38 km al E de Tziscao, 7-9 km al E. de Amparo Agua Tinta sobre el camino de Montebello a Bonapak, leg.: E Téllez \& J. L. Villasenor 6631. (XAL). - Chiapas, Mpio Ocosingo, $1.5 \mathrm{~km}$ al SE de Lacanjá-Chansayab, alt. 400 m, leg.: M. Gonzalez-Espinosa et al. 1391. (MEXU, XAL). - Chiapas, Mpio Ocosingo, 1.5 km al SW de Lacanjá-Chansayab, alt. 400 m, leg.: M. Gonzalez-Espinosa et al. 1436. (MEXU, XAL). - Chiapas, Mpio Ocosingo, a $15 \mathrm{~km}$ al NO de Boca Lacantum, camino a Palenque, alt. 220 m, leg.: E. Martinez S. 11868. (XAL). - Chiapas, Mpio Ocosingo, Crucero Corozal sobre el camino Palenque-Boca Lacantum, alt. 180 m, leg.: E. Martinez S. 18510. (MEXU, XAL). Chiapas, Mpio Ocosingo, En el vértice dek Rio Chixoy al $100 \mathrm{~km}$ al S de Boca Lacantum, camino a Chajul en zona Marquez de Comillas, alt. 120 m, leg.: E. Martinez S. 11918. (XAL). - Chiapas, Mpio Ocosingo, sobre el camino Palenque-Boca Lacantum, alt. 150 m, leg.: E. Martinez S. 17933. (XAL). - Chiapas, Mpio Ocozocoautla 660 m snm. Rancho de Cruz, camino al Rancho Corocito, leg.: J. I. Calzada 9847. (XAL 3812). - Chiapas, Mpio Ocozocoautla, El Aguacero, orillas del Rio Venta, leg.: A. Montúfar \& F. Zavala 36. (XAL). Chiapas, Mpio Oxchuc, La Cascada en el Rio Coralito, alt. 1250 m, leg.: A. Shilom Ton 5824. (MEXU, XAL). - Ibid., carr. San Cristobal a Ocosingo, leg.: A. Shilom Ton 8925. (MEXU, XAL). - Chiapas, Ray de Oxchuc, alt. 1737 m, coord.: 1653'99" N, 92²2'99” W, leg.: J. Brett 687. (XAL). - Chiapas, Mpio Sabanilla, Finca Morelia, leg.: A. Shilom Ton 7315. (MEXU, XAL). - Chiapas, Mpio Tenejapa, Arroyo Yeshab, alt. 900 m, leg.: A. Shilom Ton 5608. (MEXU, XAL). - Chiapas, Mpio Tenejapa, Cruz Pilal, leg.: A. Shilom Ton 5392. (MEXU, XAL). - Chiapas, Mpio Tila, Colonia Kikjaz, alt. 1000 m, leg.: A. Shilom Ton 5699. (MEXU, XAL). - Chiapas, Mpio Tila, Al pié del Cerro Ak'Bana, alt. 800 m, leg.: A. Shilom Ton 6002. (MEXU, XAL). - Chiapas, Mpio Tila, Finca Morelio, leg.: A. Shilom Ton 7441. (MEXU, XAL). - Chiapas, Mpio Tumbala, Cascadas de Agua Azul, orilla del arroyo, alt. 400 m, leg.: F. A. Ventura 20016. (XAL, 2 spec.). - Chiapas, Mpio Yajalón, Banco de Grava, alt. 1000 m, leg.: A. Shilom Ton 5641. (MEXU, XAL). - Chiapas, Mpio Yajalón, San Luis, leg.: A. Shilom Ton 7486. (MEXU, XAL). - Chiapas, Mpio Yajalón, Rancho San Luis, alt. 1000 m, leg.: A. Shilom Ton 8177. (MEXU, XAL). - Oaxaca, Mpio Santa Maria Jacatepec, distr. Tuxtepec, La Joya del Obispo, leg.: C. H. Ramos \& E. Martinez S. 449. (XAL). - Tabasco, Mpio Tenosique, 2.5 km arriba del cerro, campamento de la escollera a $5 \mathrm{~km}$ de Tenosique hacía E. Zapata, leg.: M. A. Magana \& S. Zamudio 833. (XAL). - Ibid., leg.: A. Shilom Ton 8925. (MEXU, XAL).

Rogiera strigosa (Benth.) Borhidi, Acta Bot. Hung. 28: 67. (1982). Basionym: Bouvardia strigosa Benth., Pl. Hartweg., p. 75. (1841). Synonym: Rondeletia strigosa (Benth.) Hemsl., Diag. Pl. Nov. Mexic., p. 27. (1879).

Specimens examined: El Salvador: Dept. La Libertad, frame of Volcán San Salvador, alt. 1800 m, leg.: L. O. Williams \& A. Molina R. 10623, 22.09.1946. (MO). - Volcán San Salva-

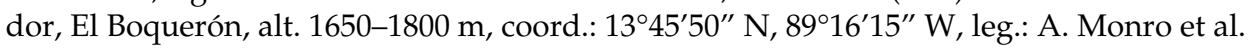
2182, 04.02.1998. (MO). - Dept. Santa Ana, Paque Nacional de Montecristo, coord.: $14^{\circ} 25^{\prime}$ N, $89^{\circ} 18^{\prime}$ W, leg.: W. Berendsohn 1056. (MO). - Santa Ana, Parque Nacional Montecristo, Cordillera de Metapán, between los planes in main road to summit of mountain, coord.: $14^{\circ} 27^{\prime}$ N, 89²2' W, alt. 1900-2000 m, leg.: G. Davidse E. et al. 37249, 27.01.1998. (MO). - Dept. Sonsonante, near top of Cerro Verde, alt. 2030 m, leg.: T. B. Croat 42183, 30.07.1977. (MO). El Imposible, Dept. Ajuachapán, San Francisco Menendez, en la cima del Cerro Mishtepe, 
coord.: $13^{\circ} 49^{\prime} \mathrm{N}, 8^{\circ} 56^{\prime} \mathrm{W}$, leg.: V. Campos s. n., 14.06.1994. (MO). Guatemala: 7 miles E of Guatemala City, alt. 1800 m, leg.: W. E. Harmon 2353, 22.05.1970. (MO). - Dept. Guatemala, along the trail up Volcán Quiquilla, alt. 4000-5000 ft, leg.: K. Torque \& D. Ledoux 469, 10.08.1975. (MO). - Dept. Quetzaltenango, pacific escarpment $7 \mathrm{~km} \mathrm{~N}$ of Santa María, planta eléctrica, on hwy 9S, alt. $2000 \mathrm{~m}$, coord.: $14^{\circ} 40^{\prime} \mathrm{N}, 9^{\circ} 30^{\prime} \mathrm{W}$, leg.: K. Roe, E. de Roe \& S. Mori 748, 31.07.1965. (MO). - Dept. San Marcos, en aldea Toniná, Volcán Tacaná, sobre camino Talquián-Tonina, cima del volcán, alt. 2700 m, leg.: E. Martinez S. \& M. Ramirez 20799, 07.05.1987. (MO). - Dept. Santa Rosa, alt. 3500 ft, leg.: Heide \& Lux 03.1892. (MO). Prov. Sacatepequez, ca 4 miles of Antigua, alt. 5500 ft, leg.: G. L. Webster et al. 11814, 27.06.1962. (MO). - Verapaz, 10 miles S of Rabinal, Baja Verapaz province, leg.: V. E. Harmon \& J. D. Dwyer 3049, 12.07.1970. (MO). Honduras: Dept. El Paraíso, along creek 2 miles of Guinope, leg.: L. O. Williams \& A. Molina R. 10319, 16.08.1946. (MO). - Dept. El Paraíso, Cerro Monserrat, bosque nebuloso, ca de Yuscarán, alt. 2000 m, leg.: C. Nelson \& E. Romero 4311, 6-9.10.1977. (MO). - Distr. Francisco Morazán, roadside banks La Cumbre, on Mt. Lepaterique, alt. 1600 m, leg.: A. Molina R. \& A. R. Molina 25870, 04.10.1970. (MO). ravines al E de Texiquat rives, $1-2 \mathrm{~km}$ SW de la aldea La Aurora, coord.: $15^{\circ} 30^{\prime} \mathrm{N}, 8^{\circ} 26^{\prime} \mathrm{W}$, alt. 200-500 m, leg.: D. L. Hazlett, H. E. Brandt \& R. Zuniga 8025, 09.04.1994. (MO). Mexico: Chiapas, ca 8 miles of NE to Solistohuacán, along hwy. 195, between Villa Hermosa and Tuxtla Gutierrez, alt. 1830 m leg.: G. Davidse 9459, 10.08.1970. (MO). - Chiapas, Escuintla, Monte Ovando, leg.: E. Matuda 4152, 01.07.1940. (MEXU, MO). - Chiapas, Kan Eh, alt. 600 m, leg.: M. Perez Gómez 91, 18.11.1987. (MO). - Chiapas, Laguna Popoy, near Lago Tsiskaw, Lagunas de Montebello Nacional Park, Mpio. La Trinitaria, alt. 1300 m, leg.: D. E. Breedlove 37065, 01.09.1970. (MO). - Chiapas, Mpio. Jitotol, 7.5 miles NW of Hierba Buena, coord.: $17^{\circ} 10^{\prime} \mathrm{N}, 92^{\circ} 57^{\prime} \mathrm{W}$, alt. 5300-5700 ft, leg.: G. L. Webster 16957, 25.08.1972. (MO). Chiapas, Mpio. Pueblo Nuevo, Solistajuacán, mirador El Caminero, $13 \mathrm{~km} \mathrm{~N}$ de Pueblo Nuevo, alt. 1450 m, leg.: W. Thomas \& L. Villasenor 3637, 25.05.1985. (MO). - Chiapas, Mpio. San Andrés Larrainzal, forested summit of Chuchil Ton, NE of Bochil, alt. $2700 \mathrm{~m}$, leg.: D. E. Breedlove 26772, 03.08.1972. (MO). - Chiapas, steep moist slope near Puerto de Viento, 9 miles N of Pueblo Nuevo Solistajuacán, along road to Tapiula, Mpio of Rayón, alt. 6100 ft, leg.: D. E. Breedlove 10149, 30.05.1965. (MO). - Chiapas, Mpio. Motozintla de Mendoza, SW slope of Cerro Mozotal, $11 \mathrm{~km}$ of the junction on the road to Motozintlaen on the road to El Porvenir and Siltepec, alt. 2100 m, leg.: D. E. Breedlove \& J. Strotherr 46208, 13.10.1980. (MO). - Oaxaca, Distr. Tejuantepec, Mpio. Cueva de Humboldt, Cerro Picacho, $8.1 \mathrm{~km}$ al N de Cueva de Humboldt, alt. $1800 \mathrm{~m}$, leg.: R. C. Torres \& L. Cortés A. 8883. 30.08.1986. (MO, 2 spec.). - Veracruz, Mpio. Soncapán, Sierra de Santa Marta, al NE de Ocotal Grande, alt. 1420 m, leg.: J. H. Beaman 5433, 08.01.1972. (MO). nicaragua: Dept. Boáco, Cerro Alegre, $5 \mathrm{~km}$ a NE de San José de los Remates, coord.: 12³6' N, 8544' W, alt. 1100-1140 m, leg.: P. P. Moreno 24896, 25104, 04.12.1984. (MO, 2 spec.). - Ibid., alt. 1100-1180 m, leg.: P. P. Moreno 20228, 11.02.1983. (MO). -Dept. de Matagalpa, km 135 entre Matagalpa y Jinotega, Cerro de la Piedra de Jicote, coord.: $12^{\circ} 58$ N, $85^{\circ} 54$ W, alt. 980-1100 m, leg.: P. P. Moreno \& W. Robleto 20462, 22.02.1983. (MO). - Dept. Jinotega, Los Mollejones, alt. 1100-1300 m, coord.: $13^{\circ} 16^{\prime} \mathrm{N}, 86^{\circ} 10^{\prime} \mathrm{W}$, leg.: P. P. Moreno 8057, 10.04.1981. (MO). Dept. Jinotega, Volcán Yalí, coord.: $13^{\circ} 16^{\prime}$ N, 86 $11^{\prime}$ W, alt. 1500-1542 m, leg.: P. P. Moreno 7954, 7977, 09.04.1981. (MO, 2 spec.). - Dept. Madríz, San José de Cuzmapa, 3 km al E del pueblo, proximo al Cerro Buena Vista, coord.: $13^{\circ} 17^{\prime} \mathrm{N}, 86^{\circ} 36^{\prime} \mathrm{W}$, alt. 1250-1300 m, leg.: P. P. Moreno 14285, 04.01.1982. (MO). 
Rogiera subscandens (Lundell) Borhidi, Acta Bot. Hung. 35: 311. (1989). Basionym: Rondeletia subscandens Lundell, Wrightia 5: 328. (1976).

Specimen examined: Mexico: Chiapas, Mpio Ocosingo, a $2 \mathrm{~km}$ al $\mathrm{N}$ de Naja, camino a Chancala, alt. 900 m, leg.: E. Martinez \& M. A. Soto A. 18762. (XAL).

Rogiera suffrutescens (Brandegee) Borhidi, Acta Bot. Hung. 28: 67. (1982). Basionym: Rondeletia suffrutescens Brandegee, Univ. Calif. Publ. Bot. 6: 70. (1914).

Specimens examined: Mexico: Chiapas, Cerro del Boquerón, leg.: C. A. Purpus 6704, 08.1913. (MO, phototype). - Chiapas, Monte Ovando, leg.: E. Matuda, 14.11.1939. (MEXU, MO). - Chiapas, hills of Union Juárez, coord.: $15^{\circ} 06^{\prime} \mathrm{N}, 92^{\circ} 05^{\prime} \mathrm{W}$, alt. $1700-2300 \mathrm{~m}$, leg.: J. S. Miller, J. K. Myers \& E. Martinez 2674, 03.05.1987. (MO).

Rondeletia L., Sp. Pl. 1: 172. (1753). Lectotype: Rondeletia americana L., designated by R. A. Howard.

Capsule depressed globose, loculicidally dehiscent, seeds fusiform, winged, caudate at one or both ends with central insertion, the testa shallowly reticulate with elongate cells; perpendicular walls simple, thin, horizontal ones densely ornamented by wall-like reticulate thickening, without globose elements. Orifice of the corolla with a conspicuous thickened, often discolourous annular callosity; corolla tube glabrous within, corolla lobes $4-5(-7)$, calyx lobes $4-5$, disc densely hirsutulous, projecting above the sinus at the base of calyx lobes, calyx tube not developed or obsolete, stipule inconspicuous, triangular or deltoid; pubescence of hypanthium, outer surface of the corolla or vegetative parts usually strigulous, neither pannose nor arachnoid pubescent; style mostly pilose at the base, placenta semiglobose, thick, horizontally sulcate, with a punctifom insertion to the septum. Pollen grain elliptic, 3-colporate, foveolate. Seed oblong, flattened, bicaudate or fusiform with central insertion, cells with thin perpendicular walls densely ornamented by globose thickenings. Basic chromosome number: $\mathrm{x}=11$.

About 140 species in the West Indies and $\mathrm{N}$ of South America, with only 1 species from Mexico and Belize to Guatemala, one in Costa Rica and Panama, and further one in Panama. Of the 85 native species recognized and listed in the Nomenclator by Lorence as valid "Rondeletia" species, not less than 82 belong to other genera. Real Rondeletia species are the following: 
Rondeletia belizensis Standl., Carnegie Inst. Wash. Publ. 461: 91. (1935). Synonym: Arachnothryx belizensis (Standl.) Borhidi, Acta Bot. Hung. 28: 70. (1982).

Specimens examined: Belize: Distr. Toledo, Solomon Camp, vicinity of the junction of Richardson Creek and Blooden Branch, foothills of the Maya Mts, coord.: $16^{\circ} 32^{\prime} \mathrm{N}, 88^{\circ} 45^{\prime}$ W, alt. 80-420 m, limestone hills. Leg.: G. Davidse \& A. E. Brandt 32144, 05.03.1987. (MO). British Honduras: Jacinto hills, alt. 300 ft, leg.: W. A. Schipp 1201, 28.08.1933. (MO, type). Guatemala: Dept. Alta Verapaz, Rubelsanto el Zapotal about 2-3 km SEE of Balastrera, leg.: C. L. Lundell \& E. Contreras 19545, 20.07.1975. (MO, type of var. longiloba Lundell). - El Petén, al E de km 179, in low forest on rocky hill, leg.: E. Contreras 10054, 10056, 14.07.1970. (MO, 2 spec.). - El Petén, Guayacán, La Pita, in low forest on rocky hill, bordering Laguna Guayacán, leg.: E. Contreras 7473, 16.01.1968. (MO). - El Petén, La Cumbre in Zapotal junto Pofhill km 141-142 of Campinas road, leg.: C. L. Lundell \& E. Contreras 19277, 10.05.1975. (MO). - El Petén, La Cumbre in Zapotal, W of the village, top of hill, on Satural road, about 6 km, leg.: C. L. Lundell \& E. Contreras 19866, 18.09.1975. (MO). - El Petén, La Cumbre,

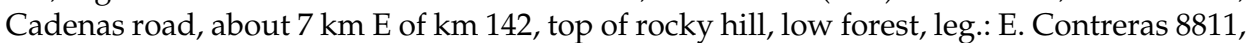
31.07.1969. (MO). - El Petén, La Cumbre, W of km 142-143 in Zapotal, on top of hill, $500 \mathrm{~m}$, leg.: C. L. Lundell \& E. Contreras 19824, 09.09.1975. (MO). - El Petén, La Libertad and vicinity, leg.: M. Aguilar 28.08.1933. (MO, with fl.!). - El Petén, La Libertad, leg.: C. L. Lundell 2854, 14.04.1933. (MO, with fr.!). - El Petén, Zeamay in low forest on top of rocky hill, about $3 \mathrm{~km}$ from the village, leg.: E. Contreras 6706, 10.03.1967. (MO). - Izabal, Puerto Mendez/Cadenas, top of rocky hill bordering the village, leg.: E. Contreras 978, 08.07.1970. (MO). Mexico: Chiapas, Mpio. Ocosingo, El Crucero Corozal sobre el camino Palenque-Boca Lacantum, alt. 400 m, leg.: E. Martinez S. \& M. H. Soto 18845, 18.06.1986. (MO).

Rondeletia deamii (Donn.Sm.) Standl., N. Amer. Fl. 32: 60. (1918). Basionym: Bouvardia deamii Donn.Sm., Bot. Gaz. 49: 455. (1910). Synonym: Arachnothryx deamii (Donn.Sm.) Borhidi, Acta Bot. Hung. 28: 70. (1982).

Specimens examined: El Salvador: Dept. Ajuachapán, El Imposible, San Francisco Menendez,Yote el Pepeto, calle a Rio San Francisco, coord.: $13^{\circ} 49^{\prime}$ N, $89^{\circ} 56^{\prime}$ W, leg.: I. Martinez s. n., 06.03.1994. (MO). - vicinity of la Union del Departamento Unio, alt. $150 \mathrm{~m}$, leg.: P. C. Standley 20656, 13.02.1922. (MO). Guatemala: Rio de las Navajas, about 14 miles site of Fiscal, alt. $3700 \mathrm{ft}$, leg.: C. C. Deam. (MO, type, Donnell Smith 6190). - roadside of the montane forest zone, $10 \mathrm{~km}$ NE of Motufar, leg.: W. A. Harmon \& J. A. Dwyer 3067, 15.07.1970. (MO). - al $6 \mathrm{~km} \mathrm{~S}$ de Quesaltepeque Chiquimala, leg.: W. E. Harmon \& J. A. Dwyer 3717, 12.08.1970. (MO). - Dept. Progreso, a $2 \mathrm{~km}$ al N de los Leones, camino el Rancho-Cobán, alt. 700 m, leg.: E. Martinez F. et al. 23223, 04.08.1988. (MO). - Ibid., leg.: E. Martinez et al. 23239. (MO). Honduras: Aguas arriba del rio faldes del Rio Majicarán, alt. 950 m, leg.: A. Molina R. 1528, 10.11.1948. (MO). - Dept. de Copán, Yaragua Creek, 1 mile W of Copán Ruinas, alt. 500 m, leg.: A. Molina R. 3861, 09.08.1975. (MO). - Dept. de Copán, Copa Maya Ruins, alt. 600 m, leg.: A. Molina R. 32102, 02.01.1983. (MO). - Dept. de Comayagua, Chichipates, orilla del Rio Yure, $30 \mathrm{~km}$ al E del Lago Yojoa, leg.: C. Nelson et al. 22.11.1980. (MO). - Dept. de Comayagua, La Ceiba, orilla del Rio Yure, $15 \mathrm{E}$ del Lago Yojoa, alt. 200 m, leg.: C. Nelson et al. 7330, 7392A, 1981.02.06 . (MO, 2 spec.). - Dept. de 
Comayagua, Vado Alto, orilla del Rio Sulaco, $33 \mathrm{~km}$ al E y $4 \mathrm{~km}$ de N de San Juan Cruz de Yojoa, alt. $150 \mathrm{~m}$, leg.: C. Nelson et al. 7644, 08.02.1984. (MO). - Dept. de Cortés, orilla del Rio Humuya, bosque de vega tropical, $40 \mathrm{~km}$ al $\mathrm{N}$ de Santa Cruz de Yojoa, alt. $100 \mathrm{~m}$, leg.: C. Nelson et al. 5751, 5831. (MO, 2 spec.). - Dept. de Yoro, camino de la ciudad de Yoro a la montana Buenos Aires, leg.: C. Nelson \& J. Martinez 1988, 28.03.1974. (MO). - Dept. El Paraíso, along the River Lisapa, between galeras and Lisapa Grande, alt. $1000 \mathrm{~m}$, leg.: A. Molina R. 171, 24.07.1947. (MO). - Dept. Ocotepeque, Forest del Cerro Chiriquí de San Antonio, alt. 1300 m, leg.: A. Molina R. 24275, 30.08.1968. (MO). - Dept. Olancho, along Rio Olancho, W of main road Tegucigalpa-Catacám hwy, ca $1 \mathrm{~km}$ upstream NW of Puente Boquerón, 8.6 miles SW of Catacamas, Santa Maria del Real, alt. $400 \mathrm{~m}$, coord.: $11^{\circ} 45^{\prime} \mathrm{N}$, 860' W, leg.: T. B. Croat \& D. P. Hammel 64127, 04.02.1987. (MO). - Dept. San Francisco Morazán, alt. 900-1000 m, quebrada al E de Cedros, leg.: C. Nelson \& E. Vargas 3508. (MO). Mexico: Edo, Oaxaca, Arroyo de las Minas, al O del Limón, entrando por el desvio a Buenos Aires, distr. Tehuántepec. leg.: R. C. Torres \& A. García 6830, 17.07.1985. (MO). nicaragua: Dept. de Chinandega, $1 \mathrm{~km}$ al S de Swanto Tomás de Nance, sobre la carretera de Somotillo. Coord.: $13^{\circ} 10^{\prime} \mathrm{N}, 8^{\circ} 55^{\prime} \mathrm{W}$, alt. 100-140 m, leg.: P. P. Moreno 11845, 30.09.1981. (MO). Dept. de Estelí, ca $3.9 \mathrm{~km}$ of $\mathrm{S}$ from Hwy road to Estanzuela, coord: $13^{\circ} 02^{\prime} \mathrm{N}, 86^{\circ} 21^{\prime} \mathrm{W}$, alt. 1000-1035 m, leg.: W. D. Stevens \& B. A. Krukoff 9938. (MO). - Dept. de León, Hacienda La Pelona, El Porvenir, Cerro de Pelona, a $2.5 \mathrm{~km}$ al NO de la casa Hacienda, alt. 500-600 m, leg.: A. Grijalva, D. Sosa \& O Estrada 4189. (MO). - Dept. de León, Malpaisillo faldas del Cerro Negro, alt. 100-200 m, leg.: M. Castro 118, 08.05.1984. (MO). - Dept. de Nueva Segovia, ca $12.3 \mathrm{~km}$ SE to Susukayán, along road to Quilalí, quebrada El Caracol. Coord.: $13^{\circ} 38^{\prime}$ N, $86^{\circ} 06^{\prime}$ W, alt. 530 m, leg.: W. D. Stevens \& B. A. Krukoff 17649, 28.07.1980. (MO). Dept. Esteli, 6 km del Pueblo Nuevo, carretera a Limay, Cerro San Ramón, coord.: $13^{\circ} 21^{\prime} \mathrm{N}$, 86³1' W, alt. 810 m, leg.: P. P. Moreno 3096, 27.09.1980. (MO). - Dept. Estelí, a km 57 a carretera de Panamericana a Santa Rosa, coord.: $13^{\circ} 11^{\prime}$ N, $86^{\circ} 21^{\prime}$ W, alt. 830 m, P. P. Moreno 21809. (MO). - Dept. Estelí, camino a Rodeo Grande, alt. 950 m, leg: A. Laguna 323, 18.01.1984. (MO). - Dept. Estelí, Entrada de San Nicolás, Las Camaras, coord.: $12^{\circ} 59^{\prime} \mathrm{N}$, $86^{\circ} 18^{\prime}$ W, alt. 1035 m, leg.: P. P. Moreno 22577, 19.12.1983. (MO). - Dept. Estelí, La Calabaza, leg.: A. Laguna 360, 12.07.1984. (MO). - Dept. Estelí, La Estanzuela, $6.5 \mathrm{~km}$ al S de Estelí, coord.: $13^{\circ} 01^{\prime} \mathrm{N}, 86^{\circ} 05^{\prime} \mathrm{W}$, alt. $1000 \mathrm{~m}$, leg.: P. P. Moreno 24389. (MO). - Dept. Estelí, La Gavilana, coord.: $13^{\circ} 03^{\prime}$ N, $86^{\circ} 19^{\prime}$ W, alt. 950-1000 m, leg.: P. P. Moreno 21878, 02.08.1983. (MO). - Ibid., alt. 1000 m, leg.: P. P. Moreno 22801, 14.01.1984. (MO). - Dept. Estelí, Mpio. San Juan de Limay, Hacienda La Grecia, Cerro Quiniento, coord.: $13^{\circ} 11^{\prime}$ N, $86^{\circ} 35^{\prime}$ W, alt. 518 m, leg.: P. P. Moreno 2141, 02.09.1980. (MO). - Dept. Estelí, Salto de Estanzuela, coord.: $13^{\circ} 01^{\prime} 30^{\prime \prime}$ N, 85²1' W, alt. 110 m, leg.: A. Grijalva \& N. B. Sandino 3301, 1986. (MO). - Dept. Estelí, subida hacía El Chayote, $23 \mathrm{~km} \mathrm{~N}$ de Estelí, coord.: $13^{\circ} 16^{\prime} \mathrm{N}, 86^{\circ} 20^{\prime} \mathrm{W}$, leg.: P. P. Moreno 21262, 01.04.1983. (MO). - Dept. Estelí, thickets along Estanzuela Creek, 108 km W de Estelí, alt. 1000 m, leg.: A. Molina R. 23083, 03.11.1960. (MO). - Dept. Matagalpa, Cerro El Nancital, SW de Santa Rosa del Penón, carretera a Santa Bárbara, coord.: $12^{\circ} 46^{\prime} \mathrm{N}, 86^{\circ} 21^{\prime} \mathrm{W}$, alt. 300-400 m, leg.: P. P. Moreno 21562, 22.06.1983. (MO, with fl.!). - Dept. Nueva Segovia, bosque de galería en el Rio Tipilto E de ciudad Ocotal, coord.: $13^{\circ} 37^{\prime} \mathrm{N}, 86^{\circ} 27^{\prime}$ W, leg.: J. C. Sandino 2969, 15.05.1982. (MO). - Dept. Nueva Segovia, ca 233.5 km, $6.5 \mathrm{~km}$ al N cerca de Ocotal, Quebrada El Nancital $13^{\circ} 41^{\prime}$ N, $86^{\circ} 24^{\prime}$ W, alt. 700-760 m, leg.: W. D. Stevens \& B. A. Krukoff 3043, 07.08.1977. (MO). - Dept. Nueva Segovia, El Jícaro, casas viejas, coord.: $13^{\circ} 44^{\prime}$ N, 86ํ5ㄴ W, alt. ca 600m, leg.: P. P. Moreno 13532, 24.12.1982. (MO). - Dept. Nueva Segovia, La Tranquera, coord.: $13^{\circ} 44^{\prime} \mathrm{N}, 8^{\circ} 04^{\prime} \mathrm{W}$, alt. $600 \mathrm{~m}$, leg.: P. P. Moreno 13716, 26.12.1981. MO). - Ibid., alt. 660-700 m, leg.: P. P. Moreno 19454, 22.12.1982. (MO). - Faldas del Chay- 
ote, coord.: $13^{\circ} 16^{\prime} \mathrm{N}, 86^{\circ} 20^{\prime} \mathrm{W}$, alt. 700-1000 m, leg.: P. P. Moreno 21785, 23.07.1983. (MO). Hacienda La Varela, $17 \mathrm{~km}$ desde el camino a Sauce, coord.: $13^{\circ} 01^{\prime} \mathrm{N}, 86^{\circ} 29^{\prime} \mathrm{W}$, alt. $445 \mathrm{~m}$, leg.: P. P. Moreno 14437, 17.09.1982. (MO).

Rondeletia hameliifolia Dwyer et Hayden, Ann. Mo. Bot. Gard. 54: 144. (1967). Synonym: Arachnothryx hameliifolia (Dwyer et Hayden) Borhidi, Acta Bot. Hung. 28: 70. (1982).

Specimens examined: Costa Rica: Cantón de la Cruz, Parque Nacional Santa Rosa,

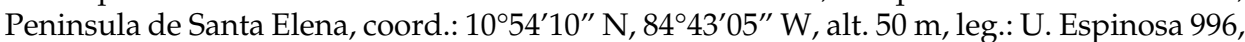
03.12.1993. (MO, 2 spec.). - Prov. Guanacaste, Santa Rosa National Park, along river Rio Guapote, alt. 100-200 m, coord.: 1050' N, 85³6’ W, leg.: R. L. Liesner 4521, 24.01.1978. (MO). Panama: Prov. Herrera, carretera Las Minas-Teze, alt. ca $0 \mathrm{~m}$, coord.: $8^{\circ} 02^{\prime} \mathrm{N}, 80^{\circ} 27^{\prime}$ W, leg.: C. Galdames et al. 1802, 29.09.1994. (MO). - Prov. Herrera, roadside, ca $3 \mathrm{~km} \mathrm{~N}$ of Las Minas, alt. 100-600 m, leg.: W. G. D'Arcy \& K. Sitsma 14283, 19.01.1983. (MO). - Prov. Los Santos, leg.: J. D. Dwyer 2458, 08.10.1962. (MO). - Prov. Los Santos, Los Asientos, leg.: C. Vendejaque 38, 15.08.1969. (MO). - Prov. Los Santos, Playa Venando, $30 \mathrm{~km} \mathrm{E} \mathrm{of}$ Tonosijon hwy 50, leg.: B. Hammel 5482, 30.10.1978. (MO). - Prov. Veraguas, carretera hacía Alto de Piedra, orilla del camino, leg.: E. Montenegro 1042, 14.08.1994. (MO). - Prov. Veraguas, Isla Coiba, Distrito de Montijo, Isla Canales de Afuera, leg.: C. Galdames, B. Araúz \& A. Castillo 3215, 24.07.1996. (MO, with fl.!). - Prov. Veraguas, Islas Contreras, Isla Brincaneo, along coast, alt. $0 \mathrm{~m}$, coord.: $7^{\circ} 51^{\prime} \mathrm{N}, 8^{\circ} 47^{\prime} \mathrm{W}$, leg.: H. W. Churchill 5721, 20.07.1984. (MO). - Prov. Veraguas, Michintole en Santiago, 50 miles from Santiago, down embankment to rivers edge, leg.: J. H. Kirkbride 164, 09.08.1967. (MO). - Prov. Veraguas, Santiago, 4 miles from the hwy to Atalaya, leg.: J. D. Dwyer \& J. H. Kirkbride 7410, 31.07.1967. (MO). - Prov. Veraguas, Santiago, 2 miles W of Santiago on Panamaerican hwy, leg.: J. D. Dwyer, S. M. W. Hayden \& J. H. Kirkbride 7556, 06.08.1967. (MO). - Prov. Veraguas, Santiago, leg.: J. D. Dwyer 1350, 08.1961. (MO, holotype). - Prov. Veraguas, US Army Tropical Center, Canazas, leg.: E. L. Tyson 3638, 26.02.1966. (MO). - Prov. Veraguas, Uva Highland of the Contreras Islands, leg.: R. Foster 1579, 25.08.1970. (MO). - Prov. Veraguas, vicinity of La Mesa, leg.: E. Hell 6057, 28.12.1968. (MO). - Prov. Veraguas, vicinity of Ponuga, leg.: J. D. Dwyer \& J. H. Kirkbride 7443, 31.07.1967. (GH, MO). - Prov. Veraguas, Distr. de Montillo, Isla Coiba, N de la Isla, ensenada Muniz, leg.: C. Galdames et al. 2139, 21.02.1995. (MO).

Rondeletia odorata Jacq. var. breviflora Hook. f., Curtis Bot. Mag. 104, tab. 6350. (1878). The type specimen (in K!) is collected in the garden of the Count of Northumberland as a cultivated plant, with short teratological flowers. Therefore, the plant is to be considered as an illegitimate taxon, as it was published by Fernandez and Herrera (1983).

Rondeletia panamensis DC., Prodr. 4: 408. (1830).

Acknowledgement - The author is highly thankful to Peter H. Raven, director of the Missouri Botanical Garden, and J. Solomon, curator of the Herbarium, to prof. Mario Sosa, 
director of MEXU, and to Sergio Guevara, director of the Ecological Institute of Mexico in Xalapa, for the permission of studying the collections and libraries of their Institutes respectively. The study has been supported by the Hungarian Scientific Research Fund (OTKA T016666 and T032036).

\section{BIBLIOGRAPHY}

Borhidi, A. (1982): Studies in Rondeletieae (Rubiaceae) III. The genera Rogiera and Arachnothryx. - Acta Bot. Hung. 28: 65-71.

Borhidi, A. (1987): Studies in Rondeletieae (Rubiaceae) X. New combinations of Central American taxa. - Acta Bot. Hung. 33: 301-303.

Borhidi, A. (1989): Studies in Rondeletieae (Rubiaceae) XI. Critical notes on some Central American species of Rondeletia s.l. - Acta Bot. Hung. 35: 309-312.

Borhidi, A. (1995): Studies in Rondeletieae XII. New combinations of Mexican and Central American taxa. - Acta Bot. Hung. 38: 139-142. (1993-94).

Borhidi, A. (1999): Studies in Rondeletieae (Rubiaceae) XIII. New combinations of Mexican and Central American plants. - Acta Bot. Hung. 40: 15-16. (1996-97).

Borhidi, A. and Darók, J. (2000): Seed coat morphology in the Rondeletia-complex. - Poster abstracts, Symposium "Flora of the Greater Antilles 2000", New York Bot. Gard., 22-25 June, 2000, pp. 9-11.

Borhidi, A. and Fernandez M. Z. (1995a): Nuevas especies y revisión breve del género Stenostomum C. F. Gaertn. (Rubiaceae) en Cuba. - Acta Bot. Hung. 38: 143-156. (1993-94).

Borhidi, A. and Fernandez M. Z. (1995b): The genus Stenostomum C. F. Gaertn. (Rubiaceae) or the reconsideration of the New World Antirhea species. - Acta Bot. Hung. 38: 157-165. (1993-94).

Borhidi, A. and Járai-Komlódi, M. (1983): Studies in Rondeletieae (Rubiaceae), IV. A new genus: Javorkaea. - Acta Bot. Hung. 29: 13-27.

Borhidi, A., Darók, J. and Kaposvári, F. (1997): The variability range of the genus Javorkaea Borhidi \& J. Komlódi (Rubiaceae). - Abstracts, IXth Hung. Plant Anat. Symp., Szeged, p. 20.

Borhidi, A., Darók, J. and Kaposvári, F. (1999): Two new species of the genus Javorkaea Borhidi et J. Komlódi (Rubiaceae). - Acta Bot. Hung. 40: 17-20. (1996-97).

Castillo-Campos, G. and Lorence, D. H. (1985): Antirhea aromatica (Rubiaceae, Guettardeae) a new species from Veracruz, Mexico. - Ann. Mo. Bot. Gard. 72: 268-271.

Chaw, S-M. and Darwin P. S. (1992): A systematic study of the paleotropical genus Antirhea (Rubiaceae, Guettardeae). - Tulane Stud. Zool. Bot. 28: 25-118.

Darók, J. (2000): A glossary of plant cuticle terminology in Rubiaceae. - Acta Bot. Hung. 42: 65-77.

Fernandez, M. Z. (1995): Estudio taxonómico del género Rondeletia L. s. 1. (Rubiaceae) en Cuba. - Acta Bot. Hung. 38: 47-138. (1993-94).

Fernandez, M. Z. and Herrera, P. O. (1983): Studies in Rondeletieae (Rubiaceae), VI. Estudio taxonómico de la Rondeletia odorata Jacq. - Acta Bot. Hung. 29: 35-41.

Lorence, D. H. (1991) New species and combinations in Mexican and Central American Rondeletia (Rubiaceae). - Novon 1: 135-157. 
Lorence, D. H. (1994): New species in Mexican and Mesoamerican Rubiaceae. - Novon 4: 119-136.

Lorence, D. H. (1997): New taxa, a new name, and a new combination in Rubiaceae from Southern Mexico and Mesoamerica. - Novon 7: 46-58.

Lorence, D. H. (1999): A Nomenclator of Mexican and Central American Rubiaceae. - Mo. Bot. Garden Press 73: 1-177.

Lorence D. H. and Castillo-Campos, G. (1988): Tres nuevas especies y una nueva combinación en el género Rondeletia (Rubiaceae, Rondeletieae) de Veracruz y Oaxaca, México. - Biotica 13: 147-157.

Standley, P. C. (1918): Rondeletia. - In: Flora of North America 32A: 44-86.

Steyermark, J. A. (1967): Rondeletia and Arachnothrix. - In: Maguire B. et al. (eds): Botany of the Guyana Highland. Part VII. Rubiaceae. Mem. N. Y. Bot. Gard. 17: 241-261. 